\title{
Dynamical Analysis of a Beddington-DeAngelis Interacting Species System with Prey Harvesting
}

\author{
Lakshmi Narayan Guin $\mathbb{D}^{1},{ }^{1}$ Reeta Murmu, ${ }^{2}$ Hunki Baek $\left(\mathbb{D},{ }^{3}\right.$ and Kyoung-Hwan Kim ${ }^{4}$ \\ ${ }^{1}$ Department of Mathematics, Visva-Bharati University, Santiniketan 731 235, West Bengal, India \\ ${ }^{2}$ Integrated Science Education and Research Center (ISERC), Visva-Bharati University, Santiniketan 731 235, West Bengal, India \\ ${ }^{3}$ Department of Mathematics Education, Daegu Catholic University, Gyeongsan, Gyeongbuk 38430, Republic of Korea \\ ${ }^{4}$ Department of International Healthcare Administration, Daegu Catholic University, Gyeongsan, \\ Gyeongbuk 38430, Republic of Korea
}

Correspondence should be addressed to Hunki Baek; hkbaek@cu.ac.kr

Received 26 June 2020; Accepted 24 September 2020; Published 28 October 2020

Academic Editor: Rosa M. Benito

Copyright (c) 2020 Lakshmi Narayan Guin et al. This is an open access article distributed under the Creative Commons Attribution License, which permits unrestricted use, distribution, and reproduction in any medium, provided the original work is properly cited.

\begin{abstract}
A reaction-diffusion interacting species system with Beddington-DeAngelis functional response that has been proposed in the environment of mathematical ecology, which provides the rise to spatial pattern formation, is investigated and associated with the models of deterministic dynamics. The dynamical behaviour of a generalist predator-prey system with linear harvesting of each species and predator-dependent functional response is fully analyzed. Conditions of stability behaviour of the interior equilibrium point are established properly. Furthermore, we have recognized that the unique positive equilibrium point of the system is globally stable via appropriate Lyapunov function structure, which signifies that appropriate harvesting has no impact on the persistence property of the harvesting system. Also, we establish the conditions for the existence of bifurcation phenomena including a saddle-node bifurcation and a Hopf bifurcation. Subsequently, complete analysis regarding the impact of harvesting is carried out, and an interesting decision is that under some appropriate constraints, harvesting has immense impact on the final size of the interacting species. In addition, in accordance with Turing's ideas on morphogenesis, our analysis shows that harvesting effort in a reaction-diffusion predator-prey system plays a vital function for geological conservation of interacting species. Finally, we discuss sufficient conditions for the existence of bionomic equilibrium point and the optimal harvesting policy attained by using the Pontryagin maximal principle.
\end{abstract}

\section{Introduction}

There has been great attention during the last few decades in dynamical features of population models and along with these models, predator-prey systems [2-6] play an imperative function in population dynamics. The dynamical correlation between predators and preys has been a vital topic in theoretical ecology since the well-known Lot$\mathrm{ka}-$ Volterra interacting species system. The vital elements in these forms of models are the predator functional response on prey species per unit time for given sizes (densities) of prey and predator. Different structures of functional responses have become the focus of considerable attention from time to time in ecological literature, for instance, preydependent functional response, predator-dependent functional response, ratio-dependent functional response, etc. To originate a more rational ecological system, an appropriate and correct variety of functional response is important.

In the current literature, usually, there are two main types of predators, namely, generalist and specialist. Specialist predators feed more or less entirely on one prey species. However, the generalist predator is rather special from specialist predator. Generalist predators feed on various categories of prey species. Once a focal prey species is exposed to extinction, the predator species is capable of changing its food habit to a different species and may 
prolong to persist; accordingly, their dynamics is not controlled by the activities of a particular prey species [7-10].

Mathematical ecology aims at the mathematical depiction, behaviour, and modelling of complex ecosystems using a choice of applied mathematical techniques and tools to get a better understanding of the system [3, 9, 11-13]. Predator-prey relationship plays a significant role in regulating the numbers of prey and predators in the environment of complex ecosystem $[14,15]$. The harvesting used by both the species has a strong impact on the system dynamics of the predator-prey interaction.

In ecology, to maintain the persistence of the species or the coexistence of the species, there are different feasible techniques such as appropriate amount of harvesting policy, constructing reserved zones or refuges, etc., which should be taken in order to help the species grow in the regions without annoyances. The functions of such kind of measures were investigated by different eminent scholars such as suitable harvesting policy in predator-prey dynamics which attracts the major attention of the researchers. By constructing a suitable Lyapunov function, some scholars proposed that the coexistence equilibrium point of the system is globally stable which reflects on the event that suitable harvesting has no influence on the persistence property of the harvesting system, whereas under some appropriate parametric restrictions, harvesting has no influence on the final size of the prey species but the size of the predator species is strictly decreasing function of the harvesting efforts [10, 16-18].

Some eminent evolutionary biologists and theoretical ecologists have recognized that the interactions between predator and their preys should not be simply explained by direct predation through prey-dependent functional responses $[16,19-21]$ and that the importance of predatordependent functional responses should be considered $[18,22,23]$; so far our knowledge goes, no mathematical models have been projected to investigate whether or the extent to which harvested generalist predator-prey model with Beddington-DeAngelis type functional response can affect interacting populations. In this investigation, the interactions of two populations have been proposed and analyzed incorporating harvesting phenomena on both species to explore the effect that harvesting can have on reaction-diffusion population dynamics in generalist predator-prey systems. Our goal in this research is to investigate the spatiotemporal dynamics of a harvested reaction-diffusion system. We develop the existence of limit cycle through Hopf bifurcation theorem and spatial patterns through diffusion-driven instability, by using the harvesting coefficient as a parameter of bifurcation.

In the present investigation, we have categorized the research work as follows. In Section 2, we propose the basic assumptions and then accordingly formulated the mathematical model. With the nondimensional model, we checked for system's (2) existence, boundedness, dissipativity, and permanence. In Section 3, we look for equilibria, and their dynamical behaviours are well studied herein. Conditions for stability are achieved and phase portraits are plotted numerically. System (2) is found to have Hopf bifurcation and saddle-node bifurcation about coexistence equilibrium point. Following in Section 4, the effect of diffusion is reflected on the system and the related pattern formation is investigated. In Discussion section, we have talked about bionomic equilibrium and optimal harvesting policy. Finally, the paper ends with a brief conclusion and significance of the obtained results in Section 6.

\section{Mathematical Models}

Assuming the importance of harvesting on a generalist predator-prey model with Beddington-DeAngelis functional response, an effort is prepared to study the influence of linear harvesting of each species in a two-dimensional (2D) Beddington-DeAngelis predator-prey model which is a modification of the following model investigated extensively by Haque [24] and Sarwadi et al. [25].

$$
\begin{aligned}
& \frac{\mathrm{d} u}{\mathrm{~d} t}=r_{1} u\left(1-\frac{u}{K_{1}}\right)-\frac{a u v}{b+u+c v}-H_{1} u, \\
& \frac{\mathrm{d} v}{\mathrm{~d} t}=r_{2} v\left(1-\frac{v}{K_{2}}\right)+\frac{e a u v}{b+u+c v}-H_{2} v, \\
& u(0) \geq 0, v(0) \geq 0,
\end{aligned}
$$

where $u(t)$ and $v(t)$ represents the population sizes of prey and generalist predator, respectively, at any instant of time $t$, and other system parameters, viz., $r_{1}, r_{2}, K_{1}, K_{2}, e, a, b, c, H_{1}, H_{2}$ are all positive. Here, $r_{1}$ and $r_{2}$ are the intrinsic growth rates of the prey and predator, respectively; $K_{1}$ and $K_{2}$ are the environmental carrying capacities of the prey and predator, respectively; $e$ is the conversion factor of prey to predator; $a$ is the maximum consumption rate and the parameter $b$ is a normalization coefficient that relates the sizes of the predator and prey to the environment in which they interact; $c$ scales the impact of the predator interference; $c<0$ is the case where predators benefits from cofeeding; $H_{1}$ and $H_{2}$ are the linear harvesting rate of prey and predator species, respectively, which means that the number of individuals harvested per unit of time is proportional to the current population $\left(0 \leq H_{1}<r_{1}, 0 \leq H_{2}<r_{2}\right)$. The predator species consumes the prey with functional response of Beddington-DeAngelis type $a u v / b+u+c v$ and contributes to its growth with $e a u v / b+u+a v[26,27]$. Throughout the investigation, the environment is believed to be uniform; that is, the ecological parameters do not depend on space and time.

Using nondimensional variables such as $\tilde{u}=u / K_{1}$, $\widetilde{v}=v / K_{2}$, and $\widetilde{t}=\mathrm{ta}$, the corresponding model can be written in dimensionless form as (dropping tildes)

$$
\begin{aligned}
& \frac{\mathrm{d} u}{\mathrm{~d} t}=u(1-u)-\frac{\varepsilon u v}{\beta+u+\alpha v}-\delta u \equiv F_{1}(u, v), \\
& \frac{\mathrm{d} v}{\mathrm{~d} t}=\gamma v(1-v)+\frac{\lambda u v}{\beta+u+\alpha v}-\sigma v \equiv F_{2}(u, v), \\
& u(0) \geq 0, v(0) \geq 0,
\end{aligned}
$$


where $\alpha=c K_{2} / K_{1}, \quad \beta=b / K_{1}, \quad \gamma=r_{2} / r_{1}, \quad \varepsilon=a K_{2} / r_{1} K_{1}$, $\delta=H_{1} / r_{1}, \lambda=e a / r_{1}$, and $\sigma=H_{2} / r_{1}$.

Now, we study the boundedness of the solutions and dissipative behaviour of the system (2). Permanence is an important behaviour of the system in the sense that it describes long-term behaviour of the system. Analytically, a system is said to be permanent if it is dissipative; i.e., all trajectories of the system (2) initiating from the point in $I R_{+}^{2}$ ultimately lie in the fixed bounded region for sufficiently large $t$.

Lemma 1. Every solution of the system (2) exists in the interval $[0, \infty)$ and $u(t) \geq 0, v(t) \geq 0$ for all $t \geq 0$.

Proof. Since $F_{1}(u, v)$ and $F_{2}(u, v)$ are completely continuous and locally Lipschitzian on $C^{1}\left(\mathrm{IR}_{+}^{2}\right)$, the solution $(u(t), v(t))$ of (2) exits and is unique on $(0, \xi)$, where $0<\xi<+\infty$. From the system (2), we have

$$
\begin{aligned}
& u(t)=u(0) \exp \left(\int_{0}^{t}\left[(1-u(s))-\delta-\frac{\varepsilon v(s)}{\beta+u(s)+\alpha v(s)}\right] \mathrm{d} s\right) \geq 0, \\
& v(t)=v(0) \exp \left(\int_{0}^{t}\left[\gamma(1-v(s))-\sigma+\frac{\lambda u(s)}{\beta+u(s)+\alpha v(s)}\right] \mathrm{d} s\right) \geq 0,
\end{aligned}
$$

which completes the proof.

Proposition 1. All the solutions of the system (2) in $I R_{+}^{2}$ are uniformly bounded.

Proof. Define a function $\eta(t)=u(t)+(\varepsilon / \lambda) v(t)$. Differentiating $\eta(t)$ with respect to $t$, we get

$$
\begin{aligned}
\frac{\mathrm{d} \eta}{\mathrm{d} t} & =\frac{\mathrm{d} u}{\mathrm{~d} t}+\frac{\varepsilon}{\lambda} \frac{\mathrm{d} v}{\mathrm{~d} t} \\
& =u(1-u)-\delta u+\frac{\gamma \varepsilon}{\lambda}[v(1-v)]-\frac{\sigma \varepsilon}{\lambda} v .
\end{aligned}
$$

For each $\xi>0$, we have

$$
\begin{aligned}
\frac{\mathrm{d} \eta}{\mathrm{d} t}+\xi \eta & =u(1-u)-\delta u+\frac{\gamma \varepsilon}{\lambda}[v(1-v)]-\frac{\sigma \varepsilon}{\lambda} v+\xi u+\xi \frac{\varepsilon}{\lambda} v \\
& =u[1-u-\delta+\xi]+\frac{\varepsilon}{\lambda} v[\gamma(1-v)-\sigma+\xi] \\
& =u[(1-\delta+\xi)-u]+\frac{\varepsilon}{\lambda} v[(\gamma-\sigma+\xi)-\gamma v] \\
& =\frac{(1-\delta-\xi)^{2}}{4}+\frac{\varepsilon}{4 \lambda \gamma}(\gamma-\sigma+\xi)^{2} .
\end{aligned}
$$

Hence, one can find $\phi>0$, such that $(\mathrm{d} \eta / \mathrm{d} t)+\varepsilon \eta \leq \phi$. From the above, we find

$$
\eta(t) \leq e^{-\xi t} \eta(0)+\frac{\phi}{\xi}\left(1-e^{-\xi t}\right) \leq \max \left[\eta(0), \frac{\phi}{\xi}\right] .
$$

Since $u(0) \geq 0, v(0) \geq 0, \eta(0)=u(0)+(\varepsilon / \lambda) v(0)$ and letting $t \longrightarrow 0$, we have $\lim _{\text {sup }} \eta(t) \leq \phi / \xi$, which is independent of the initial condition. We observe that all the solutions of the system initiating in $I R_{+}^{2}$ eventually lie in the region $S$ defined by

$$
S=\left[(u, v) \in I R_{+}^{2}: \eta=\frac{\phi}{\xi}+\kappa\right], \quad \text { for some } \kappa>0 .
$$

Hence, the proof is complete.

Proposition 2. The system (2) is dissipative if $\gamma+\lambda>\sigma$.

Proof. By usual straightforward arguments, one can show that the solution of the system (2) always exists and keeps up positive. Since all the interacting populations and parameters associated with the system (2) are positive, for the first equation of system $(2)$, we have $(\mathrm{d} u / \mathrm{d} t) \leq u(1-u)$. Consequently, by solving, one can observe that

$$
u(t) \leq \frac{1}{((1 / u(0))-1) e^{-t}+1} .
$$

Thus, any solution of the system (2) must satisfy $\lim \sup _{t \rightarrow \infty} u(t) \leq 1$.

Now, from the second equation of the system (2), we examine that

$$
\frac{\mathrm{d} v}{\mathrm{~d} t} \leq v[\gamma(1-v)-\sigma+\lambda]
$$

Therefore, we have $\limsup _{t \rightarrow \infty} v(t) \leq((\gamma+\lambda$ $-\sigma) / \gamma)=\bar{v}$, where we denote by $\bar{v}$ a unique value of $v(t)$ which is positive if $\gamma+\lambda>\sigma$. Hence, the proof is complete.

Proposition 3. The system (2) is permanent if $(\varepsilon / \alpha)+\delta<1$ and $\gamma+\lambda>\sigma$.

Proof. From the prey equation of system (2), we have

$$
\frac{\mathrm{d} u}{\mathrm{~d} t}=u(1-u)-\frac{\varepsilon \mathrm{uv}}{\beta+u+\alpha v}-\delta u \geq u\left[(1-u)-\delta-\frac{\varepsilon}{\alpha}\right] .
$$

If $\bar{u}$ is the root of $[(1-\bar{u})-\delta-(\varepsilon / \alpha)]=0$, then again by standard comparison argument, we observe that the above inequality implies $\liminf \operatorname{in}_{t \rightarrow \infty} u(t) \leq(\alpha(1-\delta)-\varepsilon / \alpha)=\bar{u}$.

Hence, for large $t$, we have $u(t) \geq \bar{u}$.

Again, from the predator system of (2), we have

$$
\frac{\mathrm{d} v}{\mathrm{~d} t}=\gamma v(1-v)+\frac{\lambda \mathrm{uv}}{\beta+u+\alpha v}-\sigma v \geq v[\gamma(1-v)-\sigma+\lambda]
$$

If $\bar{v}$ is the positive root of the quadratic equation $[\gamma(1-v)-\sigma+\lambda]=0$, then 


$$
\bar{v}=\frac{-[\alpha(\sigma-\gamma)+\gamma(u+\beta)]+\sqrt{[\alpha(\sigma-\gamma+) \gamma(u+\beta)]^{2}-4 \gamma \alpha[\beta(\sigma-\gamma)+u(\sigma-\gamma-\lambda)]}}{2 \gamma \alpha} .
$$

Therefore, we obtain $\lim \inf _{t \rightarrow \infty} v(t) \geq \bar{v}$.

Note that $\bar{u}>0$ if $\alpha>0$ and $(\varepsilon / \alpha)+\delta<1$; again $\bar{v}>0$ if $\gamma+\lambda>\sigma$. Therefore, combining these along with Proposition 2 yields the conclusion.

Hence, the proof is complete.

\section{Equilibria and Their Stability}

This section compacts with stability and bifurcation analysis of the proposed system. First, we analyze the behaviour of the proposed system through the existence of equilibrium of the system (2). The corresponding system (2) has at most four feasible equilibria in the first quadrant $I R_{+}^{2}$ as follows:

$$
\begin{aligned}
& \text { (i) } e_{0}\left(u_{0}, v_{0}\right) \text {, } \\
& \text { (ii) } e_{1}\left(u_{1}, v_{1}\right) \text {, } \\
& \text { (iii) } e_{2}\left(u_{2}, v_{2}\right) \text {, } \\
& \text { (iv) } e_{3}\left(u_{3}, v_{3}\right) \text {, }
\end{aligned}
$$

where $u_{0}=0, v_{0}=0, u_{1}=1-\delta, \quad v_{1}=0, u_{2}=0, v_{2}=1$ $-(\sigma / \gamma), \quad u_{3}=\left(\beta+\alpha v_{3}\right)\left[\sigma-\gamma\left(1-v_{3}\right)\right] / \lambda-\sigma+\gamma\left(1-v_{3}\right)$; $0<\sigma-\gamma\left(1-v_{3}\right)<\lambda$ and $v_{3}$ are the roots of the following cubic equation:

$$
a_{0} x^{3}+a_{1} x^{2}+a_{2} x+a_{3}=0,\left(a_{0} \neq 0\right)
$$

where

$$
\begin{aligned}
a_{0}= & \alpha^{2} \lambda \gamma+\varepsilon \gamma^{2}(>0), \\
a_{1}= & 2 \varepsilon \gamma(\sigma-\lambda)-\alpha^{2} \lambda(\gamma-\sigma)-2 \varepsilon \gamma^{2}+\gamma \alpha \lambda(2 \beta-\delta+1), \\
a_{2}= & 2 \varepsilon \gamma \lambda-\alpha \lambda^{2}+\lambda \sigma \alpha-\delta \lambda \gamma \beta+\varepsilon \lambda^{2}+\varepsilon \sigma^{2}+\beta^{2} \lambda \gamma-\lambda \gamma \alpha \\
& +\lambda \gamma \beta-2 \varepsilon \sigma \lambda+\lambda^{2} \delta \alpha-2 \varepsilon \gamma \sigma+\delta \lambda \gamma \alpha+\varepsilon \gamma^{2}-2 \beta \lambda \gamma \alpha \\
& +2 \beta \lambda \sigma \alpha-\delta \lambda \sigma \alpha, \\
a_{3}= & \beta^{2} \lambda(\sigma-\gamma)+\delta \beta\left(\lambda^{2}+\gamma \lambda-\lambda \sigma\right)+\lambda \beta(\sigma-\gamma) .
\end{aligned}
$$

Using Descartes' rule of signs, one may identify that the cubic equation has one and only one positive root if any one of the following inequalities holds:

$$
\begin{aligned}
& \text { (a) } a_{1}<0, a_{2}<0, a_{3}<0, \\
& \text { (b) } a_{1}>0, a_{2}<0, a_{3}<0, \\
& \text { (c) } a_{1}>0, a_{2}>0, a_{3}<0 .
\end{aligned}
$$

As a result of the ecological significance, the main concentration has been focused on investigating the qualitative dynamics around the unique and feasible coexistence equilibrium point $e_{3}\left(u_{3}, v_{3}\right)$.

Example 1. In Figure 1, we have shown the coexistence equilibrium point marked by the green circle which is the point of intersection of the prey zero-growth isoclines and the predator zero-growth isoclines for the system (2) related to the parameter values $\varepsilon=1.0, \beta=0.0041, \alpha=0.91881$, $\delta=0.2, \gamma=0.0327, \lambda=0.15$, and $\sigma=0.1$. The prey isoclines attains the axis $u=0$ and the curve $(1-u)-(\varepsilon v /(\beta+u+$ $\alpha v))-\delta=0$ and the predator isoclines attains $v=0$ and $\gamma(1-v)+(\lambda u / \beta+u+\alpha v)-\sigma=0$.

3.1. Linearization and Stability Analysis. Let us commence the following notations. The system (2) can be written in the form

$$
\dot{X}=F(X, \varepsilon)=\left(F_{1}(u, v), F_{2}(u, v)\right)^{T},
$$

where $X=(u, v)^{T}$. Here, $X^{T}$ and $\dot{X}$, respectively, represent the transpose and the derivative with respect to $t$ of $X$. The Jacobian matrix $J \equiv D F(X, \varepsilon)$ of the system (2) at any arbitrary point $(u, v)$ is given by 


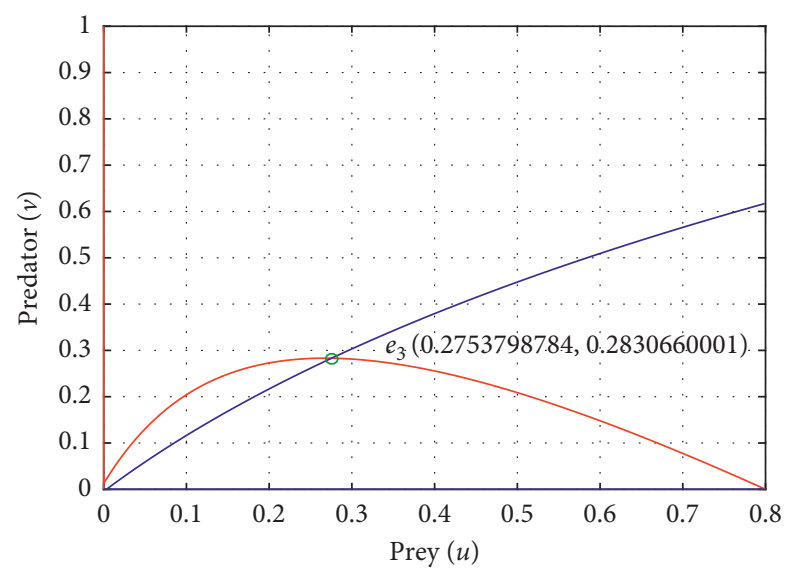

FIGURE 1: Isocline diagram of the system (2) around the positive interior point $e_{3}$ corresponding to the different parameters $\varepsilon=1.0$, $\beta=0.0041, \alpha=0.91881, \delta=0.2, \gamma=0.0327, \lambda=0.15$, and $\sigma=0.1$.

$$
\begin{aligned}
& J=\left[\begin{array}{cr}
1-2 u-\delta-\frac{\varepsilon v(\beta+\alpha v)}{(\beta+u+\alpha v)^{2}} & -\frac{\varepsilon u(\beta+u)}{(\beta+u+\alpha v)^{2}} \\
\frac{\lambda v(\beta+\alpha v)}{(\beta+u+\alpha v)^{2}} & \gamma(1-v)-\gamma v-\sigma+\frac{\lambda u(\beta+u)}{(\beta+u+\alpha v)^{2}}
\end{array}\right] \\
& =\left[\begin{array}{cc}
\frac{\varepsilon v u}{(\beta+u+\alpha v)^{2}}-u & -\frac{\varepsilon u(\beta+u)}{(\beta+u+\alpha v)^{2}} \\
\frac{\lambda v(\beta+\alpha v)}{(\beta+u+\alpha v)^{2}} & -\gamma v-\frac{\lambda u(\alpha v)}{(\beta+u+\alpha v)^{2}}
\end{array}\right]=\left[\begin{array}{ll}
\Gamma_{11} & \Gamma_{12} \\
\Gamma_{21} & \Gamma_{22}
\end{array}\right]
\end{aligned}
$$

We denote $J_{k}=J$, the Jacobian evaluated at $e_{k}, i=1,2 ; j=1,2 ; k=0,1,2,3$ and the determinant $J_{k}=\operatorname{det} J_{k}$, trace $J_{k}=\operatorname{tr}\left(J_{k}\right)$.

The dynamical behaviours of the system (2) around the equilibrium points $e_{0}, e_{1}$ and $e_{2}$ are summarized in the following theorems.

Theorem 1. The trivial equilibrium point $e_{0}(0,0)$ is

(i) saddle point if $1-\delta>0, \gamma-\sigma<0$ or $1-\delta<0, \gamma-\sigma>0$

(ii) unstable point if $1-\delta>0, \gamma-\sigma>0$,

(iii) stable point if $1<\delta$ and $\gamma<\sigma$.

Proof. The Jacobian matrix $J_{0}$ of the system (2) around the boundary equilibrium point $e_{0}(0,0)$ is given by

$$
J_{0}=\left[\begin{array}{cc}
1-\delta & 0 \\
0 & \gamma-\sigma
\end{array}\right] \text {. }
$$

The eigenvalues corresponding to $J_{0}$ are given by $1-\delta$ and $\gamma-\sigma$. Thus, one can easily identify the dynamical behaviour around the equilibrium point $e_{0}(0,0)$, via eigenvalues analysis, and accordingly the equilibrium point $e_{0}(0,0)$ is (i) saddle point if $1-\delta>0, \gamma-\sigma<0 \quad$ or $1-\delta<0, \gamma-\sigma>0$

(ii) unstable point if $1-\delta>0, \gamma-\sigma>0$

(iii) stable point if $1<\delta$ and $\gamma<\sigma$

Example 2. If one takes the parameters in the system (2) as $\varepsilon=1.0, \beta=0.0041, \alpha=0.91881, \delta=1.2, \gamma=0.0327$, $\lambda=0.15$, and $\sigma=0.1$, one can have $1<\delta$ and $\gamma<\sigma$. Thus, it follows from Theorem 1 that $e_{0}(0,0)$ is locally stable. Figure 2 shows such phenomena.

Theorem 2. The boundary equilibrium point $e_{1}(1-\delta, 0)$ is

(1) saddle point if

(i) $\delta<1$ with $\lambda+\sigma<\gamma, \beta+1<\delta$ or $\lambda+\gamma>\sigma, \beta+1>\delta$

(ii) $\delta>1 \quad$ with $\sigma>\gamma+\lambda, \beta+1<\delta \quad$ or $\gamma<\sigma+\lambda, \beta+1>\delta$

(2) unstable point if $\delta>1, \sigma<\gamma+\lambda, \beta+1<\delta$ or $\delta>1$, $\lambda+\sigma<\gamma, \beta+1>\delta$

(3) stable point if $\delta<1, \gamma<\sigma-\lambda, \beta+1>\delta$ or $\delta>1$, $\lambda+\sigma>\gamma, \beta+1<\delta$ 


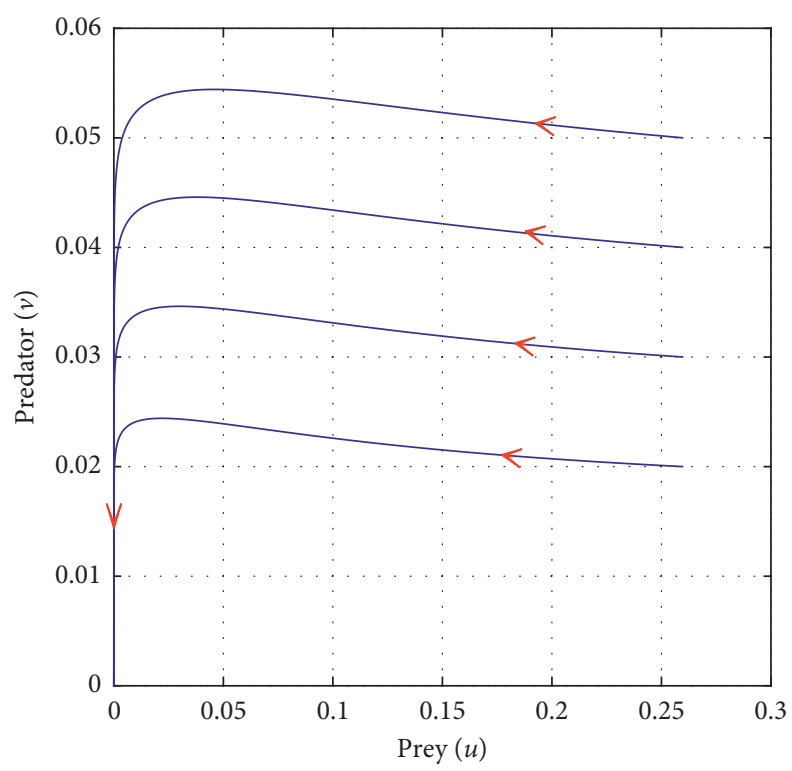

FIGURE 2: Local asymptotic stability of the system (2) around the boundary equilibrium point $e_{0}\left(u_{0}, v_{0}\right)$ corresponding to the parameters $\varepsilon=1.0, \beta=0.0041, \alpha=0.91881, \delta=1.2, \gamma=0.0327, \lambda=0.15, \sigma=0.1$ and varying the initial conditions for prey species $(u)$.

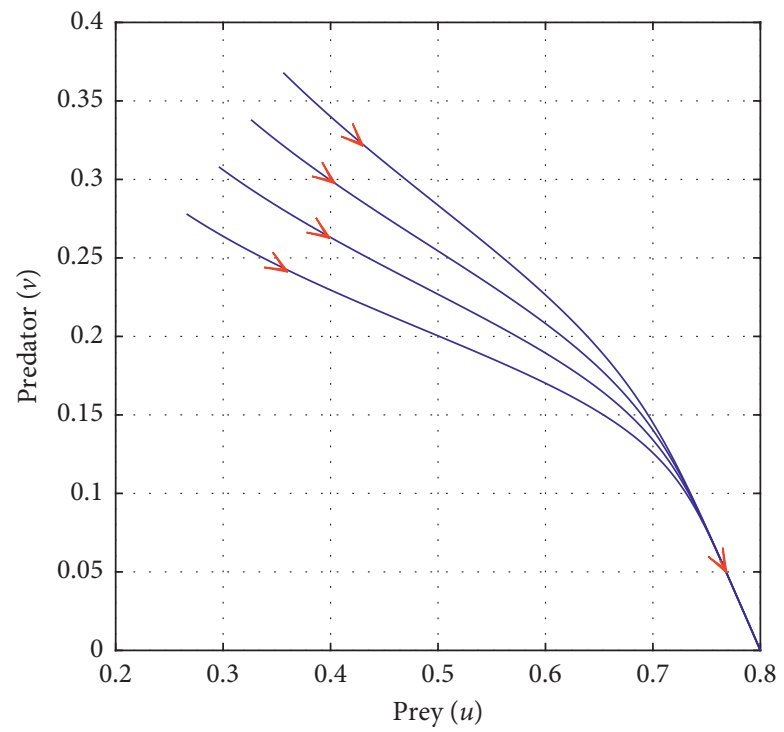

FIGURE 3: Local asymptotic stability of the system (2) around the boundary equilibrium point $e_{1}\left(u_{1}, v_{1}\right)$ corresponding to the parameters $\varepsilon=1.0, \beta=1.0041, \alpha=0.91881, \delta=0.2, \gamma=0.0327, \lambda=0.15, \sigma=0.2$ satisfying the stability condition $\delta<1, \gamma<\sigma-\lambda, \beta+1>\delta$.

Proof. The Jacobian matrix $J_{1}$ of the system (2) around the boundary equilibrium point $e_{1}(1-\delta, 0)$ is given by

$$
J_{1}=\left[\begin{array}{cc}
\delta-1 & -\frac{\varepsilon(1-\delta)}{\beta+1-\delta} \\
0 & \gamma+\frac{\lambda(1-\delta)}{\beta+1-\delta}-\sigma
\end{array}\right] .
$$

The eigenvalues corresponding to $J_{1}$ are given by $\delta-1$ and $\gamma+(\lambda(1-\delta) / \beta+1-\delta)-\sigma$. Straightforwardly, one can observe that at the equilibrium points $e_{1}(1-\delta, 0)$, the system is

(1) saddle if

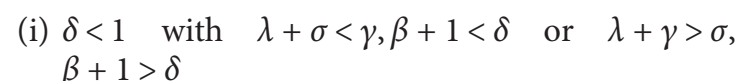


(ii) $\delta>1 \quad$ with $\quad \sigma>\gamma+\lambda, \beta+1<\delta \quad$ or $\quad \gamma<\sigma+\lambda$, $\beta+1>\delta$

(2) unstable if $\delta>1, \sigma<\gamma+\lambda, \beta+1<\delta$ or $\delta>1, \lambda+\sigma<$ $\gamma, \beta+1>\delta$

(3) stable if $\delta<1, \gamma<\sigma-\lambda, \beta+1>\delta$ or $\delta>1, \lambda+\sigma>\gamma$, $\beta+1<\delta$

Example 3. The system (2) with $\varepsilon=1.0, \beta$ $=1.0041, \alpha=0.91881, \delta=0.2, \gamma=0.0327, \lambda=0.15$, and $\sigma=$ 0.2 satisfies the conditions $\delta<1, \gamma<\sigma-\lambda$ and $\beta+1>\delta$. Thus, equilibrium point $e_{1}(1-\delta, 0)$ is locally stable. We illustrate this phenomenon in Figure 3.

Theorem 3. The axial equilibrium point $e_{2}(0,1-(\sigma / \gamma))$ is

(1) saddle point if

(i) $\sigma>\gamma \quad$ with $\quad \gamma \beta>\alpha, \gamma \beta+\varepsilon<\alpha+\delta \quad$ or $\gamma \beta<\alpha, \gamma \beta+\varepsilon>\alpha-\delta$

(ii) $\sigma<\gamma \quad$ with $\quad \gamma \beta>\alpha(\sigma-\gamma), \gamma \beta-\varepsilon>\alpha+\delta$ or $\gamma \beta<\alpha(\sigma-\gamma), \gamma \beta+\alpha<\varepsilon-\delta$

(2) unstable point if $\sigma>\gamma, \gamma \beta>\alpha, \gamma \beta+\varepsilon>\alpha+\delta$ or $\sigma>\gamma, \gamma \beta<\alpha, \gamma \beta+\varepsilon<\alpha-\delta$

(3) stable point if $\sigma<\gamma, \gamma \beta>\alpha(\sigma-\gamma), \gamma \beta+\alpha<\varepsilon+\delta$ or $\sigma<\gamma, \gamma \beta<\alpha(\sigma-\gamma), \gamma \beta+\alpha<\varepsilon-\delta$

Proof. The Jacobian matrix $J_{2}$ of the system (2) around the axial equilibrium point $e_{2}(0,1-(\sigma / \gamma))$ is given by

$$
J_{2}=\left[\begin{array}{cc}
1-\frac{\varepsilon(1-(\sigma / \gamma))}{\beta+\alpha(1-(\sigma / \gamma))}-\delta & 0 \\
\frac{\lambda(1-(\sigma / \gamma))}{\beta+\alpha(1-(\sigma / \gamma))} & -\gamma(1-(\sigma / \gamma))
\end{array}\right] .
$$

The eigenvalues corresponding to $J_{2}$ are given by $(\gamma \beta+$ $\alpha \gamma-\alpha \sigma)+\varepsilon(\sigma-\gamma)-\delta(\gamma \beta+\alpha \gamma-\alpha \sigma) / \gamma \beta+\alpha \gamma-\alpha \sigma \quad$ and $\sigma-\gamma$.
Similarly, using the same argument, one can find out that the equilibrium point $e_{2}(0,1-(\sigma / \gamma))$ is

(1) saddle point if

(i) $\sigma>\gamma \quad$ with $\quad \gamma \beta>\alpha, \gamma \beta+\varepsilon<\alpha+\delta \quad$ or $\gamma \beta<\alpha, \gamma \beta+\varepsilon>\alpha-\delta$

(ii) $\sigma<\gamma \quad$ with $\quad \gamma \beta>\alpha(\sigma-\gamma), \gamma \beta-\varepsilon>\alpha+\delta \quad$ or $\gamma \beta<\alpha(\sigma-\gamma), \gamma \beta+\alpha<\varepsilon-\delta$

(2) unstable point if $\sigma>\gamma, \gamma \beta>\alpha, \gamma \beta+\varepsilon>\alpha+\delta$ or $\sigma>\gamma, \gamma \beta<\alpha, \gamma \beta+\varepsilon<\alpha-\delta$

(3) stable point if $\sigma<\gamma, \gamma \beta>\alpha(\sigma-\gamma), \gamma \beta+\alpha<\varepsilon+\delta$ or $\sigma<\gamma, \gamma \beta<\alpha(\sigma-\gamma), \gamma \beta+\alpha<\varepsilon-\delta$

Example 4. If one chooses the parameters in the system (2) as $\varepsilon=1.0, \beta=0.0041, \alpha=0.91881, \delta=0.2, \gamma=0.1327$, $\lambda=0.15$, and $\sigma=0.1$, it is easy to see that the conditions $\delta<1, \gamma<\sigma-\lambda$ and $\beta+1>\delta$ are satisfied. Thus, from Theorem 10, one can know that the equilibrium point $e_{2}(0,1-$ $(\sigma / \gamma))$ is locally stable as shown in Figure 4.

Now we mention the dynamical behaviours of the system (2) around the interior equilibrium point $e_{3}\left(u_{3}, v_{3}\right)$ as follows.

Theorem 4. The coexistence equilibrium point $e_{3}\left(u_{3}, v_{3}\right)$ is

(i) locally asymptotically stable if $\varepsilon v_{3}<\left(\beta+u_{3}+\alpha v_{3}\right)^{2}$

(ii) If $1-\left(\varepsilon / \beta^{2}\right) v_{3}>0$, then the local stability of $e_{3}\left(u_{3}, v_{3}\right)$ ensures its global stability

Proof

(i) In order to prove that the system is locally asymptotically stable around the equilibrium point $e_{3}\left(u_{3}, v_{3}\right)$, firstly, we need to observe the Jacobian matrix $J_{3}$ of the system around the point, which is given by

$$
\begin{aligned}
J_{3} & =\left[\begin{array}{cc}
1-2 u_{3}-\delta-\frac{\varepsilon v_{3}\left(\beta+\alpha v_{3}\right)}{\left(\beta+u_{3}+\alpha v_{3}\right)^{2}} & -\frac{\varepsilon u_{3}\left(\beta+u_{3}\right)}{\left(\beta+u_{3}+\alpha v_{3}\right)^{2}} \\
\frac{\lambda v_{3}\left(\beta+\alpha v_{3}\right)}{\left(\beta+u_{3}+\alpha v_{3}\right)^{2}} & \gamma\left(1-v_{3}\right)-\gamma v_{3}+\frac{\lambda u_{3}\left(\beta+u_{3}\right)}{\left(\beta+u_{3}+\alpha v_{3}\right)^{2}}-\sigma
\end{array}\right] \\
& =\left[\begin{array}{cc}
\frac{\varepsilon u_{3} v_{3}}{\left(\beta+u_{3}+\alpha v_{3}\right)^{2}}-u_{3} & -\frac{\varepsilon u_{3}\left(\beta+u_{3}\right)}{\left(\beta+u_{3}+\alpha v_{3}\right)^{2}} \\
\frac{\lambda v_{3}\left(\beta+\alpha v_{3}\right)}{\left(\beta+u_{3}+\alpha v_{3}\right)^{2}} & -\frac{\lambda u_{3}\left(\alpha v_{3}\right)}{\left(\beta+u_{3}+\alpha v_{3}\right)^{2}}-\gamma v_{3}
\end{array}\right]=\left[\begin{array}{ll}
\Gamma_{11} & \Gamma_{12} \\
\Gamma_{21} & \Gamma_{22}
\end{array}\right] .
\end{aligned}
$$




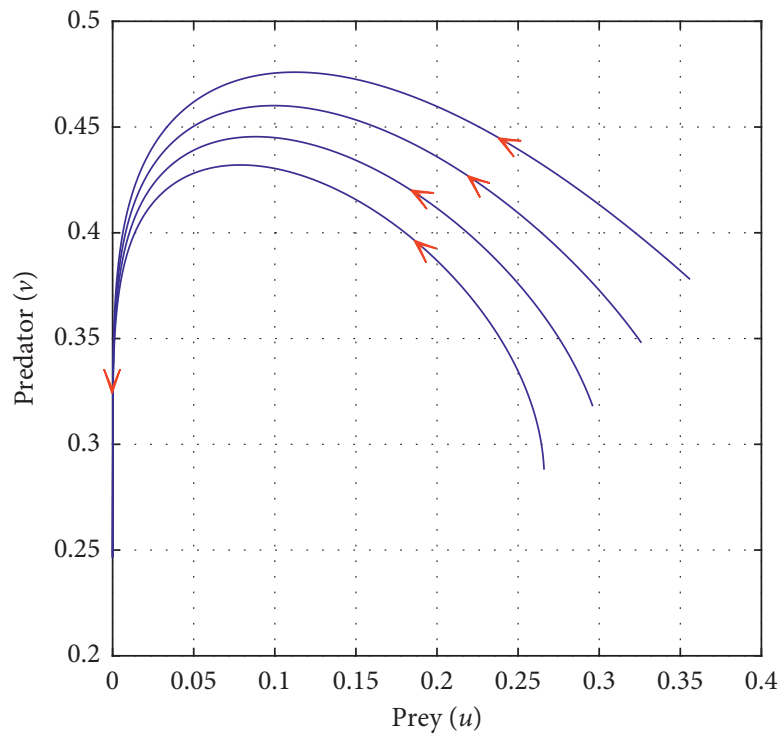

FIGURE 4: Local asymptotic stability of the system (2) around the boundary equilibrium point $e_{2}\left(u_{2}, v_{2}\right)$ corresponding to the parameters $\varepsilon=1.0, \beta=0.0041, \alpha=0.91881, \delta=0.2, \gamma=0.1327, \lambda=0.15, \sigma=0.1$ satisfying the stability condition $\sigma<\gamma, \gamma \beta>\alpha(\sigma-\gamma), \gamma \beta+\alpha<\varepsilon+\delta$.
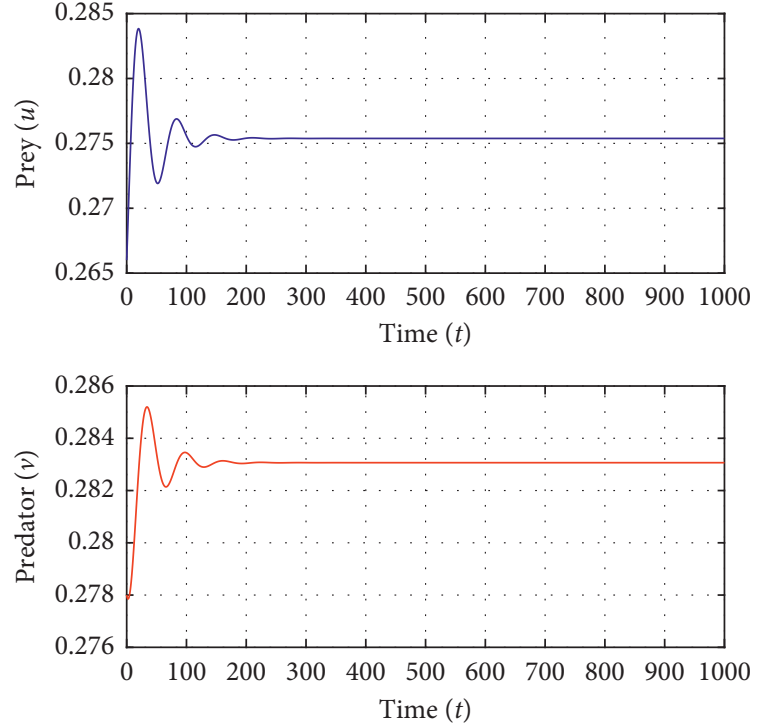

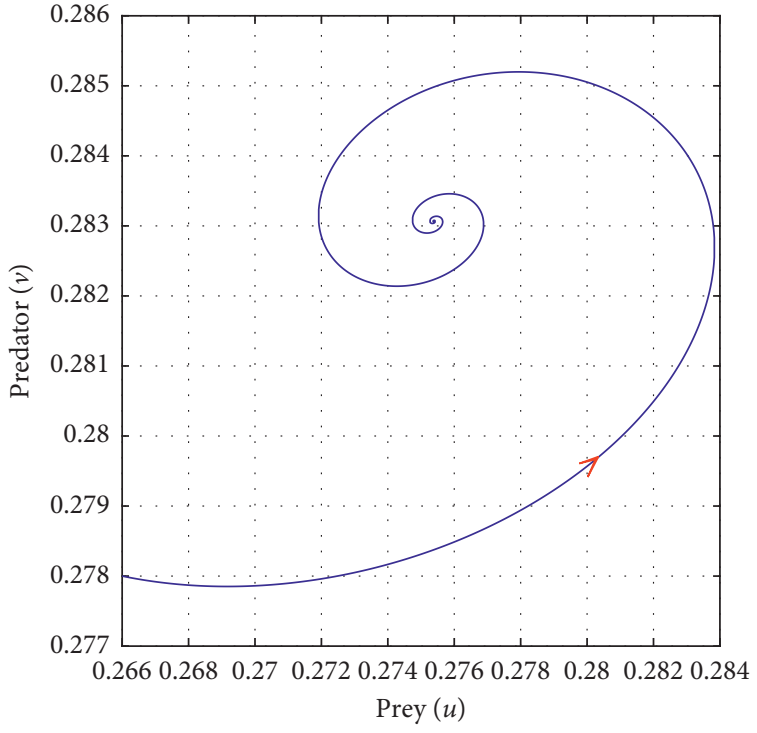

$e_{3}(0.2753798784,0.2830660001) \quad$ corresponding

FiguRE 5: Global stability of the system $\quad$ (2)
$\varepsilon=1.0, \beta=0.0041, \alpha=0.91881, \delta=0.2, \gamma=0.0327, \lambda=0.15, \sigma=0.1$.

From the Routh-Hurwitz criterion, given as tr $\left(J_{3}\right)<0$ and det $J_{3}>0$, we can get $\Gamma_{11}<0$, which gives $\varepsilon v_{3}<\left(\beta+u_{3}+\alpha v_{3}\right)^{2}$.

(ii) To show the global stability of the system (2), we construct a Lyapunov function as follows:

$$
\begin{aligned}
L(u, v) & =L_{1} \int_{u_{3}}^{u} \frac{\xi_{1}-u_{3}}{\xi_{1}} d \xi_{1}+L_{2} \int_{v_{3}}^{v} \frac{\xi_{2}-v_{3}}{\xi_{2}} d \xi_{2} \\
& =L_{1}\left(u-u_{3}-u_{3} \log \frac{u}{u_{3}}\right)+L_{2}\left(v-v_{3}-v_{3} \log \frac{v}{v_{3}}\right),
\end{aligned}
$$


where $L_{1}$ and $L_{2}$ are positive constants to be determined. Clearly, $L(u, v)=0$ at $e_{3}\left(u_{3}, v_{3}\right)$ and is positive for all other positive values of $u$ and $v$. The time derivative of $L(u, v)$ along the solution of the system (2) is

$$
\begin{aligned}
\frac{\mathrm{d} L}{\mathrm{~d} t}= & L_{1}\left(\frac{\mathrm{d} u}{\mathrm{~d} t}-\frac{u_{3}}{u} \frac{\mathrm{d} u}{\mathrm{~d} t}\right)+L_{2}\left(\frac{\mathrm{d} v}{\mathrm{~d} t}-\frac{v_{3}}{v} \frac{\mathrm{d} v}{\mathrm{~d} t}\right) \\
= & L_{1}\left(\frac{u-u_{3}}{u}\right) \frac{\mathrm{d} u}{\mathrm{~d} t}+L_{2}\left(\frac{v-v_{3}}{v}\right) \frac{\mathrm{d} v}{\mathrm{~d} t} \\
= & L_{1}\left(\frac{u-u_{3}}{u}\right)\left[u(1-u)-\delta u-\frac{\varepsilon u v}{\beta+u+\alpha v}\right]+L_{2}\left(\frac{v-v_{3}}{v}\right)\left[\gamma v(1-v)-\sigma v+\frac{\lambda u v}{\beta+u+\alpha v}\right] \\
= & L_{1}\left(u-u_{3}\right)\left[(1-u)-\delta-\frac{\varepsilon v}{\beta+u+\alpha v}\right]+L_{2}\left(v-v_{3}\right)\left[\gamma(1-v)-\sigma+\frac{\lambda u}{\beta+u+\alpha v}\right] \\
= & L_{1}\left(u-u_{3}\right)\left[u_{3}+\frac{\varepsilon v_{3}}{\beta+u_{3}+\alpha v_{3}}-u-\frac{\varepsilon v}{\beta+u+\alpha v}\right]+L_{2}\left(v-v_{3}\right)\left[\gamma v_{3}-\frac{\lambda u_{3}}{\beta+u_{3}+\alpha v_{3}}-\gamma v+\frac{\lambda u}{\beta+u+\alpha v}\right] \\
= & L_{1}\left(u-u_{3}\right)\left[-\left(u-u_{3}\right)+\frac{\varepsilon}{(\beta+u+\alpha v)\left(\beta+u_{3}+\alpha v_{3}\right)}\left(\beta v_{3}+u v_{3}-v \beta-v u_{3}\right)\right] \\
& +L_{2}\left(v-v_{3}\right)\left[-\gamma\left(v-v_{3}\right)+\frac{\lambda}{(\beta+u+\alpha v)\left(\beta+u_{3}+\alpha v_{3}\right)}\left(\beta u+\alpha u v_{3}-u_{3} \beta-\alpha v u_{3}\right)\right] \\
\leq & L_{1}\left(u-u_{3}\right)\left\{-\left(u-u_{3}\right)+\frac{\varepsilon}{\beta^{2}}\left[\beta\left(v_{3}-v\right)+\left(u v_{3}-v u_{3}\right)\right]\right\}+L_{2}\left(v-v_{3}\right)\left\{-\gamma\left(v-v_{3}\right)+\frac{\lambda}{\beta^{2}}\left[\beta\left(u-u_{3}\right)+\alpha\left(u v_{3}-v u_{3}\right)\right]\right\} \\
= & -L_{1}\left(u-u_{3}\right)^{2}-L_{1}\left(u-u_{3}\right) \frac{\varepsilon}{\beta}\left(v-v_{3}\right)+L_{1}\left(u-u_{3}\right) \frac{\varepsilon}{\beta^{2}}\left(u v_{3}-v u_{3}\right)-\gamma L_{2}\left(v-v_{3}\right)^{2}+L_{2}\left(v-v_{3}\right) \frac{\lambda}{\beta}\left(u-u_{3}\right) \\
& +L_{2}\left(v-v_{3}\right) \frac{\lambda \alpha}{\beta^{2}}\left(u v_{3}-v u_{3}\right) \\
= & -L_{1}\left(u-u_{3}\right)^{2}-L_{1}\left(\frac{\varepsilon}{\beta}\right)\left(u-u_{3}\right)\left(v-v_{3}\right)+L_{1}\left(\frac{\varepsilon}{\beta^{2}}\right) v_{3}\left(u-u_{3}\right)^{2}-L_{1}\left(\frac{\varepsilon}{\beta^{2}}\right) u_{3}\left(u-u_{3}\right)\left(v-v_{3}\right) \\
& -\gamma L_{2}\left(v-v_{3}\right)^{2}+L_{2}\left(\frac{\lambda}{\beta}\right)\left(u-u_{3}\right)\left(v-v_{3}\right)+L_{2}\left(\frac{\lambda \alpha}{\beta^{2}}\right) v_{3}\left(u-u_{3}\right)\left(v-v_{3}\right)-L_{2}\left(\frac{\lambda \alpha}{\beta^{2}}\right) u_{3}\left(v-v_{3}\right)^{2} \\
= & -L_{1}\left(u-u_{3}\right)^{2}\left[1-\left(\frac{\varepsilon}{\beta^{2}}\right) v_{3}\right]-L_{2}\left(v-v_{3}\right)^{2}\left[\gamma+\left(\frac{\lambda \alpha}{\beta^{2}}\right) u_{3}\right]-\left(u-u_{3}\right)\left(v-v_{3}\right)\left[L_{1} \frac{\varepsilon}{\beta}\left(1+\frac{u_{3}}{\beta}\right)-L_{2} \frac{\lambda}{\beta}\left(1+\frac{\alpha v_{3}}{\beta^{2}}\right)\right], \\
&
\end{aligned}
$$

Choosing $L_{1}=1$ and $L_{2}=\varepsilon\left(\beta+u_{3}\right) / \lambda\left(\beta+\alpha v_{3}\right)$, one can write

$$
\begin{aligned}
& \frac{\mathrm{d} L}{\mathrm{~d} t} \leq-\left\{\left(u-u_{3}\right)^{2}\left(1-\frac{\varepsilon}{\beta^{2}} v_{3}\right)+\left(v-v_{3}\right)^{2}\right. \\
&\left.\cdot\left(\gamma+\frac{\lambda \alpha}{\beta^{2}} u_{3}\right)\left[\frac{\varepsilon\left(\beta+u_{3}\right)}{\lambda\left(\beta+\alpha v_{3}\right)}\right]\right\} .
\end{aligned}
$$

Thus, if $1-\left(\varepsilon / \beta^{2}\right) v_{3}>0$, then $\mathrm{d} L / \mathrm{d} t<0$ and $\mathrm{d} L / \mathrm{d} t=0$ if $(u, v)=\left(u_{3}, v_{3}\right)$. Hence, the proof is complete.
Example 5. Numerically, we display the solution of the system (2) with $\varepsilon=1.0, \beta=0.0041, \alpha=0.91881, \delta=0.2$, $\gamma=0.0327, \lambda=0.15$, and $\sigma=0.1$ in Figure 5. It follows from Theorem 4 that the equilibrium point $e_{3}(0.2753798784$, $0.2830660001)$ is globally stable due to $1-\left(\varepsilon / \beta^{2}\right) v_{3}>0$.

3.2. Bifurcation Analysis of the System. Now, we investigate bifurcation phenomena of the system according to the parameter value $\varepsilon$.

Theorem 5. The system (2) undergoes a saddle-node bifurcation around $e_{3}\left(u_{3}, v_{3}\right)$ at $\varepsilon=\varepsilon_{[S N]}$, where

$$
\varepsilon_{[\mathrm{SN}]}=\frac{\gamma v_{3}^{3} \alpha^{3}+3 \alpha^{2} v_{3}^{3} \gamma\left(\beta+u_{3}\right)+\alpha^{2} \lambda u_{3} v_{3}+3 \alpha v_{3} \gamma\left(\beta^{2}+\beta u_{3}+u_{3}^{2}\right)+\alpha u_{3} \lambda\left(u_{3}+\beta\right)+\beta \gamma\left(\beta^{2}+3 \beta u_{3}+3 u_{3}^{2}\right)+u_{3}^{3} \gamma}{\alpha \gamma v_{3}^{2}+\left(u_{3}+\beta\right) v_{3} \gamma-\lambda \beta} .
$$


Proof. For a saddle-node bifurcation, one of the eigenvalues of $J_{3}$ has to be zero which is possible iff det $\left(J_{3}\right)=\left(\Gamma_{11} \Gamma_{22}-\Gamma_{12} \Gamma_{21}\right)=0$, which gives $\mathcal{\varepsilon}=\varepsilon_{[\mathrm{SN}]}$. The other eigenvalue is obtained from the relation $\operatorname{tr}\left(J_{3}\right)=\Gamma_{11}+\Gamma_{22}$ evaluated at $\varepsilon=\varepsilon_{[S N]}$ and should be negative [28].

Let $\sigma$ and $\Upsilon$ be the eigenvectors corresponding to eigenvalue 0 of the matrix $\left(J_{3}\right)$ and its transpose, respectively. We obtain that $\delta=\left[\theta_{1}, \theta_{2}\right]^{T}, \Upsilon=\left[\hbar_{1}, \hbar_{2}\right]^{T}$ where

$$
\begin{gathered}
\theta_{1}=-\frac{\Gamma_{12} \theta_{2}}{\Gamma_{11}}=-\frac{\Gamma_{22} \theta_{2}}{\Gamma_{21}}, \\
\hbar_{1}=-\frac{\Gamma_{21} \hbar_{2}}{\Gamma_{11}}=-\frac{\Gamma_{22} \hbar_{2}}{\Gamma_{12}} .
\end{gathered}
$$

And $\theta_{2}, \hbar_{2}$ are any two nonzero real numbers. Now, for the system (2), we can get

$$
\begin{aligned}
& \Upsilon^{T}\left[F_{\varepsilon}\left(X_{3}, \varepsilon_{[\mathrm{SN}]}\right)\right]=\left[\hbar_{1} \hbar_{2}\right]\left[\begin{array}{l}
\frac{\partial F_{1}}{\partial \varepsilon} \\
\frac{\partial F_{2}}{\partial \varepsilon}
\end{array}\right]=\left[\hbar_{1} \hbar_{2}\right]\left[\begin{array}{c}
\frac{-u v}{\beta+u+\alpha v} \\
0
\end{array}\right]=-\hbar_{1}\left(\frac{u v}{\beta+u+\alpha v}\right) \neq 0 \\
& \Upsilon^{T}\left[D^{2} F\left(X_{3}, \varepsilon_{[\mathrm{SN}]}\right)(\widetilde{\delta}, \sigma)\right]=\left[\hbar_{1} \hbar_{2}\right]\left[\begin{array}{l}
\frac{\partial^{2} F_{1}}{\partial u^{2}} \theta_{1}^{2}+\frac{\partial^{2} F_{1}}{\partial u \partial v} \theta_{1} \theta_{2}+\frac{\partial^{2} F_{1}}{\partial v \partial u} \theta_{2} \theta_{1}+\frac{\partial^{2} F_{1}}{\partial v^{2}} \theta_{2}^{2} \\
\frac{\partial^{2} F_{2}}{\partial u^{2}} \theta_{1}^{2}+\frac{\partial^{2} F_{2}}{\partial u \partial v} \theta_{1} \theta_{2}+\frac{\partial^{2} F_{2}}{\partial v \partial u} \theta_{2} \theta_{1}+\frac{\partial^{2} F_{2}}{\partial v^{2}} \theta_{2}^{2}
\end{array}\right] \\
& =\left(\hbar_{1}\right)\left[\frac{\partial^{2} F_{1}}{\partial u^{2}} \theta_{1}^{2}+\frac{\partial^{2} F_{1}}{\partial u \partial v} \theta_{1} \theta_{2}+\frac{\partial^{2} F_{1}}{\partial v \partial u} \theta_{2} \theta_{1}+\frac{\partial^{2} F_{1}}{\partial v^{2}} \theta_{2}^{2}\right] \\
& +\left(\hbar_{2}\right)\left[\frac{\partial^{2} F_{2}}{\partial u^{2}} \theta_{1}^{2}+\frac{\partial^{2} F_{2}}{\partial u \partial v} \theta_{1} \theta_{2}+\frac{\partial^{2} F_{2}}{\partial v \partial u} \theta_{2} \theta_{1}+\frac{\partial^{2} F_{2}}{\partial v^{2}} \theta_{2}^{2}\right] \\
& =\left(\hbar_{1}\right)\left[\frac{\partial}{\partial u}\left(1-2 u-\delta-\frac{\varepsilon v(\beta+\alpha v)}{(\beta+u+\alpha v)^{2}}\right) \theta_{1}^{2}-\frac{\partial}{\partial u}\left(\frac{\varepsilon u(\beta+u)}{(\beta+u+\alpha v)^{2}}\right) \theta_{1} \theta_{2}\right. \\
& \left.+\frac{\partial}{\partial v}\left(1-2 u-\delta-\frac{\varepsilon v(\beta+\alpha v)}{(\beta+u+\alpha v)^{2}}\right) \theta_{2} \theta_{1}-\frac{\partial}{\partial v}\left(\frac{\varepsilon u(\beta+u)}{(\beta+u+\alpha v)^{2}}\right) \theta_{2}^{2}\right] \\
& +\left(\hbar_{2}\right)\left[\frac{\partial}{\partial u}\left(\frac{\lambda v(\beta+\alpha v)}{(\beta+u+\alpha v)^{2}}\right) \theta_{1}^{2}+\frac{\partial}{\partial u}\left(\gamma(1-v)-\gamma v+\frac{\lambda u(\beta+u)}{(\beta+u+\alpha v)^{2}}-\sigma\right) \theta_{1} \theta_{2}\right. \\
& \left.+\frac{\partial}{\partial v}\left(\frac{\lambda v(\beta+\alpha v)}{(\beta+u+\alpha v)^{2}} \theta_{2} \theta_{1}\right)+\frac{\partial}{\partial v}\left(\gamma(1-v)-\gamma v+\frac{\lambda u(\beta+u)}{(\beta+u+\alpha v)^{2}}-\sigma\right) \theta_{2}^{2}\right] \\
& =\left(\hbar_{1}\right)\left[\left(-2+\frac{2 \varepsilon v(\beta+\alpha v)}{(\beta+u+\alpha v)^{3}}\right) \theta_{1}^{2}-2\left(\frac{\varepsilon\left(\beta^{2}+\beta u+\beta \alpha v+2 u \alpha v\right)}{(\beta+u+\alpha v)^{3}}\right) \theta_{1} \theta_{2}+\left(\frac{2 \varepsilon \alpha u(\beta+u)}{(\beta+u+\alpha v)^{3}}\right) \theta_{2}^{2}\right] \\
& -\left(\hbar_{2}\right)\left[\left(\frac{2 \lambda v(\beta+\alpha v)}{(\beta+u+\alpha v)^{3}}\right) \theta_{1}^{2}+\left(\frac{\lambda\left(\beta^{2}+\beta u+\beta \alpha v+2 u v \alpha\right)}{(\beta+u+\alpha v)^{3}}\right) \theta_{1} \theta_{2}-\left(2 \gamma+\frac{2 u \lambda \alpha(\beta+u)}{(\beta+u+\alpha v)^{3}}\right) \theta_{2}^{2}\right] \\
& =-\left(2 \theta_{1}^{2} \hbar_{1}+2 \gamma \theta_{2}^{2} \hbar_{2}\right)+\frac{1}{(\beta+u+\alpha v)^{3}}\left\{( \hbar _ { 1 } ) \left[2 \varepsilon v(\beta+v \alpha) \theta_{1}^{2}-2 \varepsilon\left(\beta^{2}+u \beta+v \beta \alpha+2 u v \alpha\right) \theta_{1} \theta_{2}\right.\right. \\
& \left.\left.+2 u \varepsilon \alpha(\beta+u) \theta_{2}^{2}\right]-\left(\hbar_{2}\right)\left[2 v \lambda(\beta+v \alpha) \theta_{2}^{2}-2 \lambda\left(\beta^{2}+u \beta+v \beta \alpha+2 u v \alpha\right) \theta_{1} \theta_{2}-2 u \lambda \alpha(\beta+u) \theta_{2}^{2}\right]\right\} \\
& \neq 0 \text {. }
\end{aligned}
$$


Here, we write $(u, v)$ instead of $\left(u_{3}, v_{3}\right)$ for algebraic convenience.

So, the system experiences a saddle-node bifurcation [28] around the positive interior equilibrium $e_{3}\left(u_{3}, v_{3}\right)$ at $\varepsilon=\varepsilon_{[\mathrm{SN}]}$. Again, since $\Upsilon^{T}\left[F_{\varepsilon}\left(X_{2}, \varepsilon_{[\mathrm{SN}]}\right)\right] \neq 0$, therefore, the system does not attain neither transcritical bifurcation nor pitch-fork bifurcation.

Theorem 6. The system (2) admits a Hopf bifurcation around $e_{3}\left(u_{3}, v_{3}\right)$ at $\varepsilon=\varepsilon_{[H B]}$, where $\varepsilon=\varepsilon_{[H B]}=\lambda \alpha+\left(u_{3}+\gamma v_{3}\right)\left(\beta+u_{3}+\alpha v_{3}\right)^{2} / u_{3} v_{3}$.

Proof. In a system, a Hopf bifurcation typically occurs when a complex conjugate pair of eigenvalues of the linearized flow at the fixed point $e_{3}\left(u_{3}, v_{3}\right)$ becomes purely imaginary, i.e., if det $\left(J_{3}\right)>0$ and if $\operatorname{tr}\left(J_{3}\right)=0$ for some value of $\varepsilon$ say $\varepsilon_{[\mathrm{HB}]}$.

The characteristic equation is given by

$$
\mu^{2}-\left(\Gamma_{11}+\Gamma_{22}\right) \mu+\left(\Gamma_{11} \Gamma_{22}-\Gamma_{12} \Gamma_{21}\right)=0,
$$

where $u-u_{3} \approx e^{\mu t}$ and $v-v_{3} \approx e^{\mu t}$.

If $\operatorname{tr}\left(J_{3}\right)=\Gamma_{11}+\Gamma_{22}=0$ at $\varepsilon=\varepsilon_{[\mathrm{HB}]}$, then both the eigenvalues will be purely imaginary if $\operatorname{det}\left(J_{3}\right)=\Gamma_{11} \Gamma_{22}-$ $\Gamma_{12} \Gamma_{21}$ is positive.

Replacing $\mu=\mu_{1}+i \mu_{2}$ into the corresponding characteristic equation and separating real and imaginary parts, we get

$$
\begin{array}{r}
\left(\mu_{1}^{2}-\mu_{2}^{2}\right)-\operatorname{tr}\left(J_{3}\right) \mu_{1}+\operatorname{det}\left(J_{3}\right)=0 \\
2 \mu_{1} \mu_{2}-\operatorname{tr}\left(J_{3}\right) \mu_{2}=0 .
\end{array}
$$

Elementary differential of (30) with respect to $\varepsilon$ and considering $\mu_{1}=0$, we get

$$
\left.\frac{\mathrm{d} \mu_{1}}{\mathrm{~d} \varepsilon}\right]_{\varepsilon=\varepsilon_{[\mathrm{HB}]}}=\frac{v_{3} u_{3}}{2\left[\left(\beta+u_{3}+\alpha v_{3}\right)^{2}\right]} \neq 0,
$$

Hence, the system goes through a Hopf bifurcation around $e_{3}\left(u_{3}, v_{3}\right)$ at $\varepsilon=\varepsilon_{[\mathrm{HB}]}$, where

$$
\varepsilon_{[\mathrm{HB}]}=\lambda \alpha+\frac{\left(u_{3}+\gamma v_{3}\right)\left(\beta+u_{3}+\alpha v_{3}\right)^{2}}{u_{3} v_{3}} \text {. }
$$

Example 6. It follows from Theorem 6 that the system could have a limit cycle on account of Hopf bifurcation. In fact, Figure 6 shows the existence of a limit cycle numerically when the parameters are taken as $\varepsilon=1.0596222$, $\beta=0.0041, \alpha=0.91881, \delta=0.2, \gamma=0.0327, \lambda=0.15$, and $\sigma=0.1$ in the system (2).

Remark 1. For better understanding of qualitative change of the temporal dynamics of the system (2), one may observe the bifurcation diagrams of both prey and predator species with respect to the system parameter $\epsilon$ (cf. Figures 7 and 8). In support of this technique of bifurcation offered in Figure 7 , the successive maxima of $u$ and $v$ in the ranges
$[0.01,0.8]$ and $[0.01,0.33]$, respectively, as a function of $\epsilon$ in the range $0.95 \leq \epsilon \leq 1.2$ and the other system parameters are captioned in the figure. The other bifurcation diagram is generated for the successive maxima of population sizes of $u$ and $v$ in the ranges $[0.01,0.9]$ and $[0.0,0.7]$, respectively, as a function of $\epsilon$ in the range $0.8 \leq \epsilon \leq 1.5$ and the other system parameters are captioned in Figure 8. It is interesting to note from Figures 7 and 8 that maximum concentration of predator species reduces drastically in the presence of prey harvesting factor $\delta$ at positive level. Due to the certain amount of prey harvesting from the interacting species system, predator species cannot consume prey species in earlier manner and accordingly this scenario supports the natural phenomena very well.

\section{Pattern Formation Analysis of the System}

In 1952, Alan Turing recommended in his seminal work on "The Chemical Basis of Morphogenesis" that the formation of biological patterns can be understood through reaction-diffusion framework, that is, in a form of coupled system of nonlinear parabolic equations [1]. The novelty of Turing's 1952 paper has influenced many evolutionary biologists, theoretical ecologists, and mathematicians. Under this development, chemicals produced by cells interact like activators or inhibitors and diffuse through the medium at diverse rates. This can generate a symmetry breaking of the uniform concentrations, a mechanism that is frequently called a "diffusion-driven instability" [29]. Turing recommended an ingenious mathematical theory to produce spatial patterns in a chemical system. For this, he reflected on a system of morphogens reacting and diffusing in such an approach that, without diffusion, they revealed a spatially uniform steady state which would be stable. Then, he explained that the presence of diffusion could lead to diffusion-driven instability resulting in a spatially heterogeneous pattern of chemical concentrations. A reaction-diffusion interacting species model equation with harvesting of such dynamics has been offered herein to study biological pattern formation. Consequently, the system (2) develops into a reaction-diffusion predator-prey system:

$$
\begin{gathered}
\frac{\partial u}{\partial t}=u(1-u)-\frac{\varepsilon \mathrm{uv}}{\beta+u+\alpha v}-\delta u+d_{1} \nabla^{2} u \\
\frac{\partial v}{\partial t}=\gamma v(1-v)+\frac{\lambda \mathrm{uv}}{\beta+u+\alpha v}-\sigma v+d_{2} \nabla^{2} v \\
u(0, x, y) \geq 0, v(0, x, y) \geq 0
\end{gathered}
$$

where $u(0, x, y)$ and $v(0, x, y)$ stand for the prey and predator species sizes at any time $t$ and at the spatial location $(x, y)$ in $2 \mathrm{D}$ spatial domain; $\nabla^{2} \equiv\left(\partial^{2} / \partial x^{2}\right)+\left(\partial^{2} / \partial y^{2}\right)$ stands for the common Laplacian operator in 2D spatial domain $\Omega$; and the remaining system parameters have the same meaning as those mentioned in case of system (2). One may believe that the environment is uniform; that is, the system parameters are independent of space or time. 

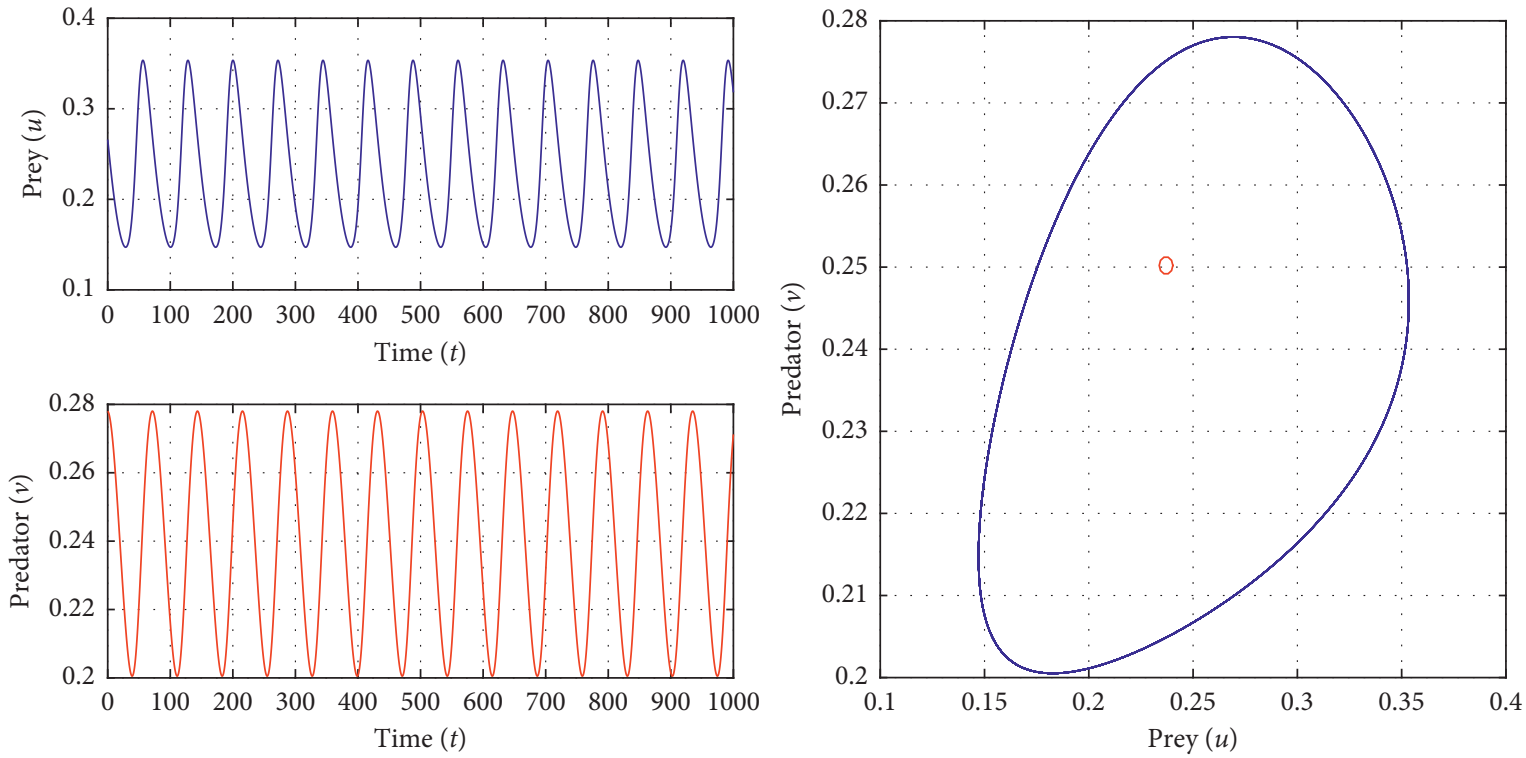

Figure 6: A limit cycle of the system (2) around $e_{3}(0.2371368969,0.2503112874)$ corresponding to $\varepsilon=1.0596222, \beta=0.0041$, $\alpha=0.91881, \delta=0.2, \gamma=0.0327, \lambda=0.15, \sigma=0.1$.
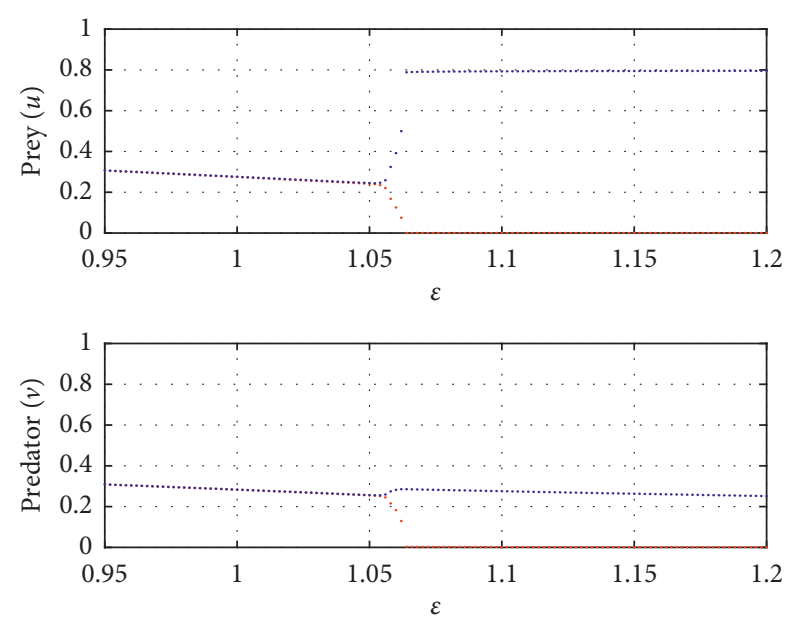

FIGURE 7: The bifurcation of the system (2) around $e_{3}\left(u_{3}, v_{3}\right)$ corresponding to the bifurcating parameter $\varepsilon$, where other parameter values are $\beta=0.0041, \alpha=0.91881, \delta=0.2, \gamma=0.0327$, $\lambda=0.15, \sigma=0.1$.

Mathematically, the offered reaction-diffusion model in this section is coupled nonlinear partial differential equations (PDEs). The parameters $d_{2}$ and $d_{1}$ are the positive selfdiffusion coefficients of the $v$ and $u$, respectively. As a general rule, self-diffusion is assumed as a spatial transmission way, which shifts from higher concentration on the way to lower concentration. Note that since the reaction-diffusion model (34a)-(34c) takes into account possible existence of alternative food sources, generalist predator can survive without prey and extinction of prey does not lead to extinction of predator species. Also, one may believe the subsequent zeroflux boundary conditions:
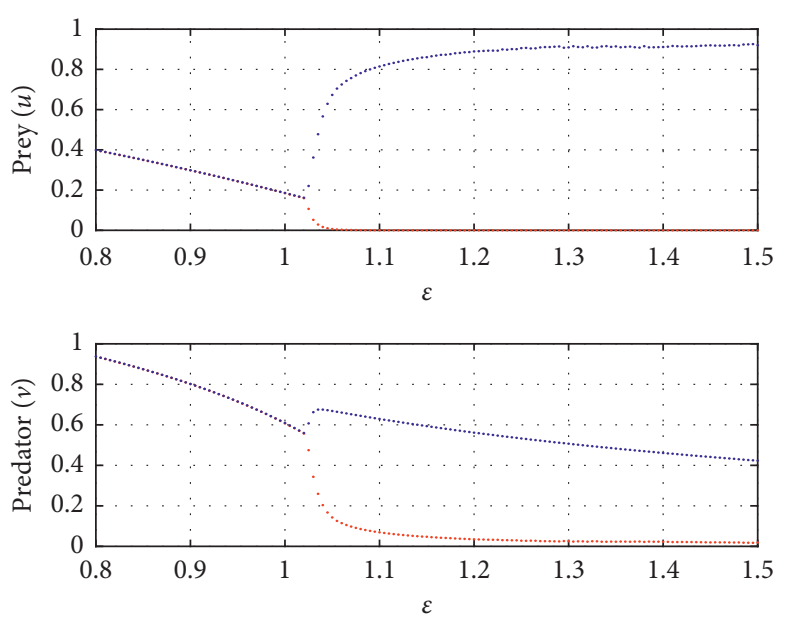

FIgURE 8: The bifurcation of the system (2) around $e_{3}\left(u_{3}, v_{3}\right)$ corresponding to the bifurcating parameter $\varepsilon$, where other parameter values are $\beta=0.031, \alpha=0.871881, \delta=0.0, \gamma=0.0327$, $\lambda=0.15, \sigma=0.05$.

$$
\frac{\partial u}{\partial n}=\frac{\partial v}{\partial n}=0,(x, y) \in \partial \Omega
$$

where $n$ is the outward unit normal vector of the smooth boundary $\partial \Omega$. The zero-flux boundary conditions signify that the system is self-contained with zero-species flux across the boundary.

Previously, we have observed that the unique and positive coexistence equilibrium point $e_{3}\left(u_{3}, v_{3}\right)$ exists under some parametric restrictions. Moreover, one may notice that the coexistence equilibrium point $e_{3}\left(u_{3}, v_{3}\right)$ for the nonspatial model of (34a)-(34c) is a spatially homogeneous steady state for the reaction-diffusion system (34a)-(34c). With definite parametric restrictions, such a steady state 
could be linearly stable without the presence of diffusion factor but linearly unstable with diffusion factor; this is the well-known recognizable information of diffusion-driven instability [1].

Linear stability analysis about the homogeneous steady state $e_{3}\left(u_{3}, v_{3}\right)$ for the reaction-diffusion system (34a)-(34c) and the essential conditions for the onset of diffusion-driven instability are well known. On a square 2D domain with zero-flux boundary conditions, diffusion-driven instability needs the following conditions (see $[3,9,10,13]$ for detailed information):

$$
\begin{aligned}
& \text { (i) } \Gamma_{11}+\Gamma_{22}<0, \\
& \text { (ii) } \Gamma_{11} \Gamma_{22}-\Gamma_{12} \Gamma_{21}>0, \\
& \text { (iii) } d_{1} \Gamma_{22}+d_{2} \Gamma_{11}>0, \\
& \text { (iv) } \frac{\left(d_{1} \Gamma_{22}+d_{2} \Gamma_{11}\right)^{2}}{4 d_{1} d_{2}}>\left(\Gamma_{11} \Gamma_{22}-\Gamma_{12} \Gamma_{21}\right), \\
& \Longrightarrow d_{1} \Gamma_{22}+d_{2} \Gamma_{11}>2 \sqrt{d_{1} d_{2}\left(\Gamma_{11} \Gamma_{22}-\Gamma_{12} \Gamma_{21}\right)},
\end{aligned}
$$

where the Jacobian matrix $J$ of the nonspatial system of $(34 a)-(34 c)$ is given by

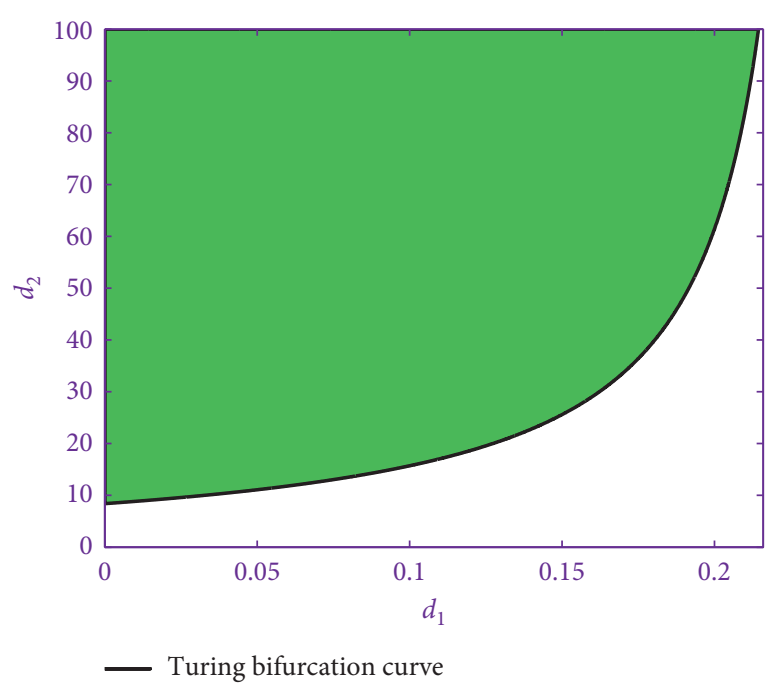

FIgure 9: Turing's space of the reaction-diffusion model (34a)-(34c) in $\left(d_{1}, d_{2}\right)$ parametric space related to the parameter values $\alpha=0.91881, \beta=0.01, \gamma=0.0327, \delta=0.05, \varepsilon=0.9, \lambda=0.5$, and $\sigma=0.05$

$$
J=\left[\begin{array}{cc}
1-2 u_{3}-\delta-\frac{\varepsilon v_{3}\left(\beta+\alpha v_{3}\right)}{\left(\beta+u_{3}+\alpha v_{3}\right)^{2}} & -\frac{\varepsilon u_{3}\left(\beta+u_{3}\right)}{\left(\beta+u_{3}+\alpha v_{3}\right)^{2}} \\
\frac{\lambda v_{3}\left(\beta+\alpha v_{3}\right)}{\left(\beta+u_{3}+\alpha v_{3}\right)^{2}} & \gamma\left(1-v_{3}\right)-\gamma v_{3}-\sigma+\frac{\lambda u_{3}\left(\beta+u_{3}\right)}{\left(\beta+u_{3}+\alpha v_{3}\right)^{2}}
\end{array}\right]=\left(\Gamma_{\mathrm{ij}}\right)_{(2 \times 2)} \quad i, j=1,2,
$$

Out of four conditions, the first two conditions of diffusion-driven instability are well-known Routh-Hurwitz criterion for which the coexistence equilibrium point $e_{3}$ related to the nonspatial system of $(34 a)-(34 c)$ is linearly stable. One may interestingly observe that the last condition (iv) summarizes condition (iii) as well. Moreover, one may examine that the three restrictions (i), (ii), (iv) are essential conditions for spatial pattern formation given the proper perturbation and system length scale [30, 31]. One may define "Turing's space" as the parametric space which satisfies the four conditions (i)-(iv) of diffusion-driven instability.

Figure 9 shows the diffusion-driven instability region of the diffusive system (34a)-(34c) in $d_{1} d_{2}$-plane related to $\alpha=0.91881, \beta=0.01, \gamma=0.0327, \delta=0.05, \varepsilon=0.9, \lambda=0.5$, and $\sigma=0.05$, and we have captured contour pictures of Turing's patterns in this region.

4.1. The Spatial Patterns through Diffusion-Driven Instability of the System (34a)-(34c). This section deals with the presentation of extensive numerical simulations of the reaction-diffusion predator-prey system (34a)-(34c) with the effect of harvesting and shows qualitative consequences in two-dimensional space. The numerical integration of the system (34a)-(34c) is executed through explicit Euler method with time step size 0.01 ; space step size 1.0; system size $200 \times 200$, and the standard five-point approximation for the two-dimensional Laplacian with zero-flux boundary conditions. The initial conditions are always a small amplitude random perturbation about $e_{3}$.

Different kinds of dynamics are examined through numerical simulation and we have figured out that the distributions of predator and prey are always of the same type. For this reason, one may confine their interest to the pattern formation of prey species or predator species only where the parameter values are in the region of Turing's space. But we have incorporated spatial patterns for predator species to understand the fluctuation of predator's concentration properly.

Figure 10 demonstrates the development of spatial pattern formation for the reaction-diffusion system (34a)-(34c) corresponding to the system parameter values, captioned in the connected figure. In this case, the pattern takes a long time to settle down, starting with a homogeneous state $e_{3}\left(u_{3}, v_{3}\right)$ and the random perturbation leads to 


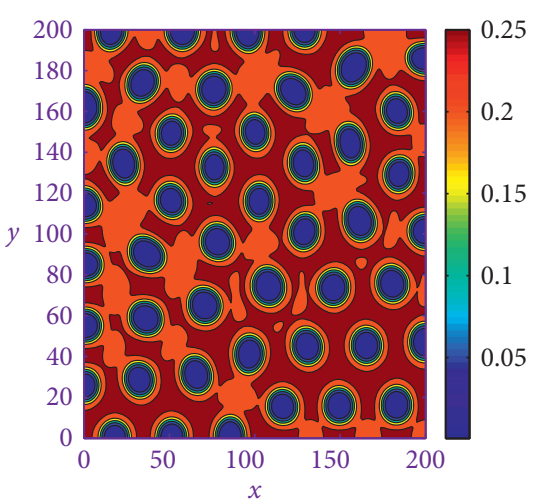

(a)

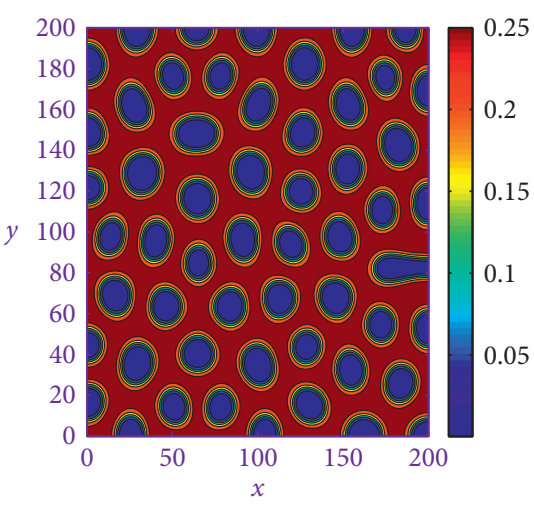

(b)

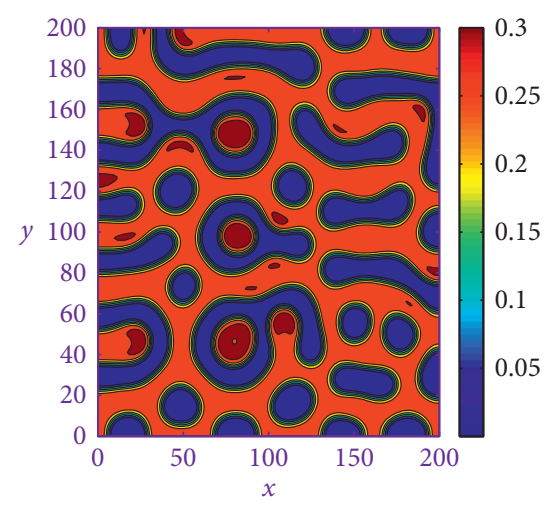

(c)

Figure 10: Numerical integration of the reaction-diffusion system (34a)-(34c) with Neumann boundaries; to generate stationary Turing's patterns by prey species $(u)$ at (a) $\delta=0.0$; (b) $\delta=0.01$; and (c) $\delta=0.023$. Other parameter values are $\alpha=0.91881, \beta=0.005, \gamma=0.0327$, $\varepsilon=1.0, \lambda=0.15, \sigma=0.05, d_{1}=0.1$, and $d_{2}=20.0$.

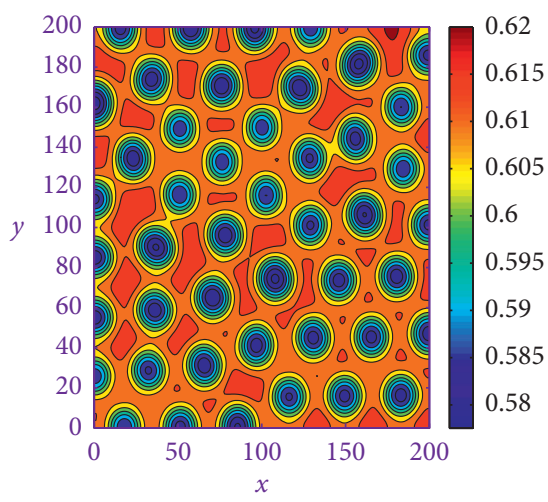

(a)

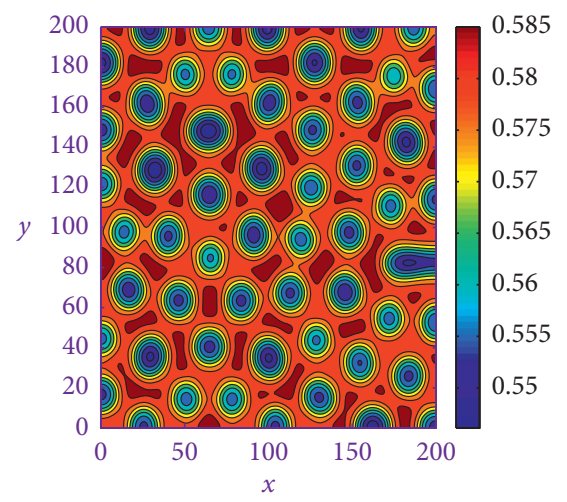

(b)

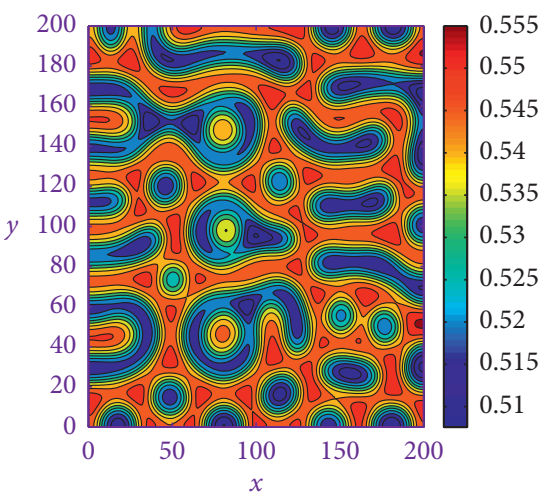

(c)

FIGURE 11: Numerical integration of the reaction-diffusion system (34a)-(34c) with Neumann boundaries; to generate stationary Turing's patterns by predator species $(v)$ at (a) $\delta=0.0$; (b) $\delta=0.01$; and (c) $\delta=0.023$. Other parameter values are $\alpha=0.91881, \beta=0.005, \gamma=0.0327$, $\varepsilon=1.0, \lambda=0.15, \sigma=0.05, d_{1}=0.1$, and $d_{2}=20.0$.

the formation of cold spots and typical circular patterns mixture (cf. Figure 10(c)) from cold spots patterns (cf. Figure 10(a)) at stationary level. Also, from Figure 11, one may identify predator spatial patterns sequence simultaneously. From the colour bar of each panel, one may notice that due to the increasing value of prey harvesting parameter $\delta$, the size/concentration of prey species is also increasing but in the other way generalist predator's size/concentration is subsequently decreasing. Biologically, one may conclude that optimal harvesting policy is essential for both species to survive in the system.

When $\delta=0.184$, we illustrate the cold spots patterns of the prey species in the spatial domain at $t=20000$ in Figure 12(a) corresponding to $\alpha=0.91881, \beta=0.0041$, $\gamma=0.0327, \quad \varepsilon=1.0, \quad \lambda=0.164, \quad \sigma=0.1, \quad d_{1}=0.1, \quad$ and $d_{2}=20.0$. Although the qualitative feature of all the figures (12(a) for $\delta=0.184,12$ (b) for $\delta=0.192$, and 12(c) for $\delta=0.2$ ) are similar, there is a fundamental difference for the spatially extended mode. At last, we find out that the cold spots patterns with smaller radius prevail over the whole spatial domain and the system dynamics does not undergo any further changes. From Figure 13, one may recognize predator spatial patterns sequence, in a similar manner.

On increasing the value of significant parameter $\delta$, one may observe the following prey spatial patterns sequence from Figure 14: "cold spots (cf. Figure 14(a)) $\longrightarrow$ labyrinthine (cf. Figure 14(b)) $\longrightarrow$ stripes (cf. Figure $14(\mathrm{c})$ ) $\longrightarrow$ hot spots-stripes mixtures (cf. Figure $14(\mathrm{~d})$ ) $\longrightarrow$ hot spots-short stripes mixtures (cf. Figure 14(e)) $\longrightarrow$ hot spots (cf. Figure 14(f))." Moreover, from Figure 15, one may detect predator spatial patterns sequence simultaneously. It is interesting to note from the colour bar of each panel that due to the increasing value of prey harvesting parameter $\delta$ from 5\% to $9 \%$, the size/concentration of prey species is also increasing but in the other approach generalist predator's size/concentration is subsequently decreasing. Biologically, one may conclude that optimal harvesting policy is essential for both species to survive in the system. 


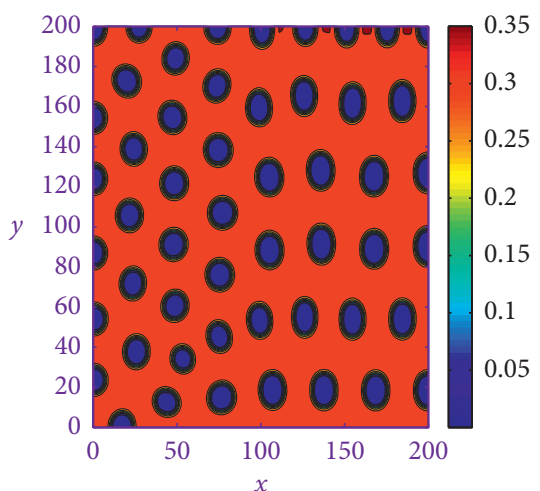

(a)

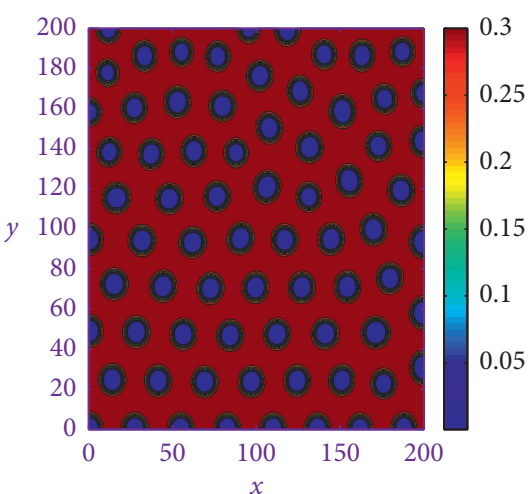

(b)

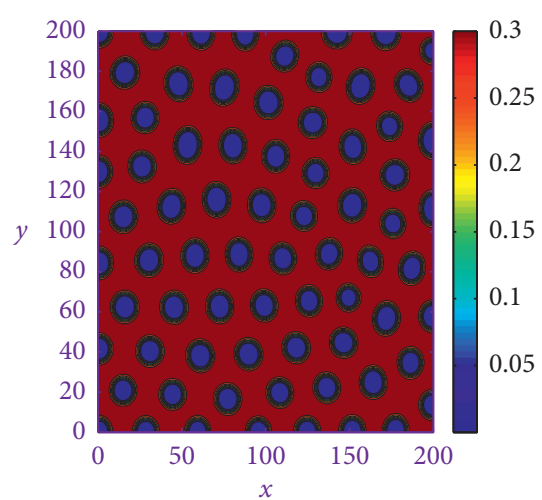

(c)

Figure 12: Numerical integration of the reaction-diffusion system (34a)-(34c) with Neumann boundaries; to generate stationary Turing's patterns by prey species $(u$ ) at (a) $\delta=0.184$; (b) $\delta=0.192$; and (c) $\delta=0.2$. Other parameter values are $\alpha=0.91881, \beta=0.0041, \gamma=0.0327$, $\varepsilon=1.0, \lambda=0.164, \sigma=0.1, d_{1}=0.1$, and $d_{2}=20.0$.

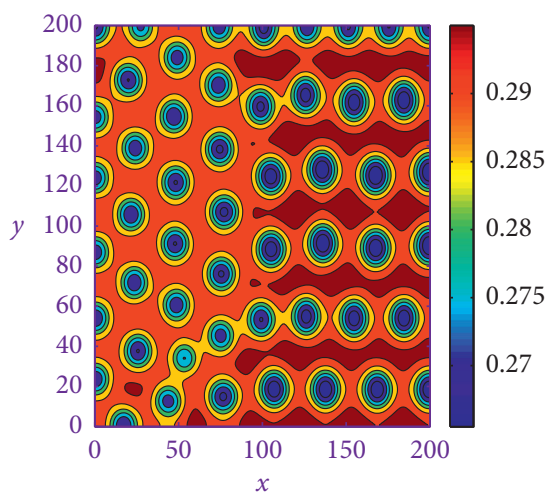

(a)

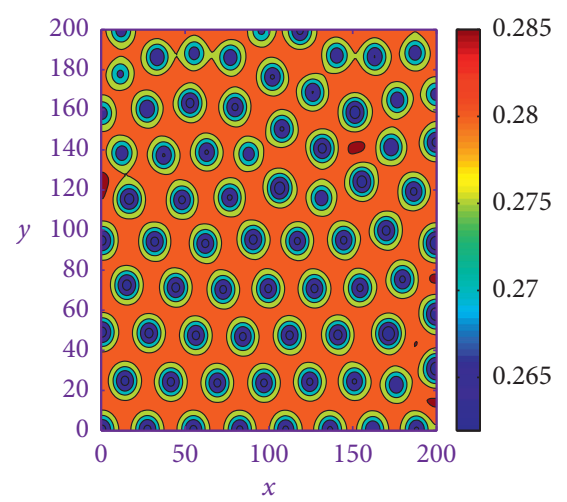

(b)

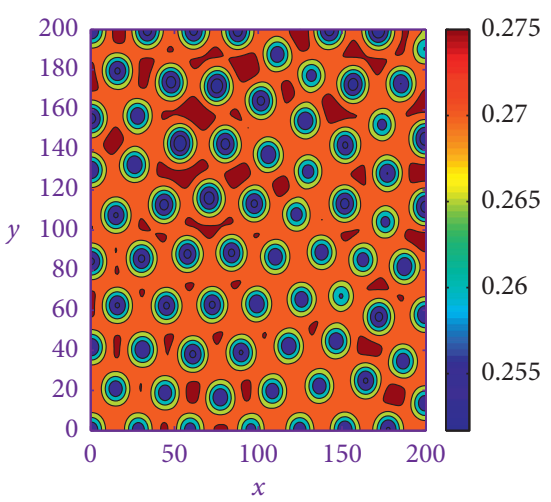

(c)

FIGURE 13: Numerical integration of the reaction-diffusion system (34a)-(34c) with Neumann boundaries; to generate stationary Turing's patterns by predator species (v) at (a) $\delta=0.184$; (b) $\delta=0.192$; and (c) $\delta=0.2$. Other parameter values are $\alpha=0.91881, \beta=0.0041$, $\gamma=0.0327, \varepsilon=1.0, \lambda=0.164, \sigma=0.1, d_{1}=0.1$, and $d_{2}=20.0$.

However, if we fix the harvesting coefficient for prey species $(\delta)$ at some positive level, then for increasing values of harvesting coefficient for predator species $\sigma$ at positive level, the numerical simulation develops to a mixed-mode steady state whose graphical depiction is not presented here for the sake of brevity.

\section{Discussion}

In this section, we discuss optimal harvesting problems and bionomic equilibrium of the system (2). In fact, until now, although there are many researches dealt with predator-prey system with harvesting (cf. [10, 21, 32-37]), it is not prevalent to study optimal harvesting strategy and economic cost theoretically.

5.1. Bionomic Equilibrium. The term is derived from the concept concerning with both biological and economic equilibrium and is given by $(\mathrm{d} u / \mathrm{d} t)=(\mathrm{d} v / \mathrm{d} t)=0$. The economic equilibrium is said to be achieved when the $T R$ (total revenue collected by selling the harvested species) equals $T C$ (the total cost for the effort devoted for harvesting).

The revenue at any time $t$ (economic rent) is given by

$$
N=\mathrm{TR}-\mathrm{TC}=\left(p_{1} u-q_{1}\right) \delta+\left(p_{2} v-q_{2}\right) \sigma,
$$

where $p_{1}=$ price per unit biomass of the prey species $(u)$, $p_{2}=$ price per unit biomass of the predator species $(v)$, $q_{1}=$ fishing cost per unit effort of the prey species $(u)$, and $q_{2}=$ fishing cost per unit effort of the predator species $(v)$.

So, the bionomic equilibrium is given by the simultaneous equations:

$$
\begin{aligned}
& \dot{u}=u(1-u)-\frac{\varepsilon \mathrm{uv}}{\beta+u+\alpha v}-\delta u, \\
& \dot{v}=\gamma v(1-v)+\frac{\lambda \mathrm{uv}}{\beta+u+\alpha v}-\sigma v
\end{aligned}
$$




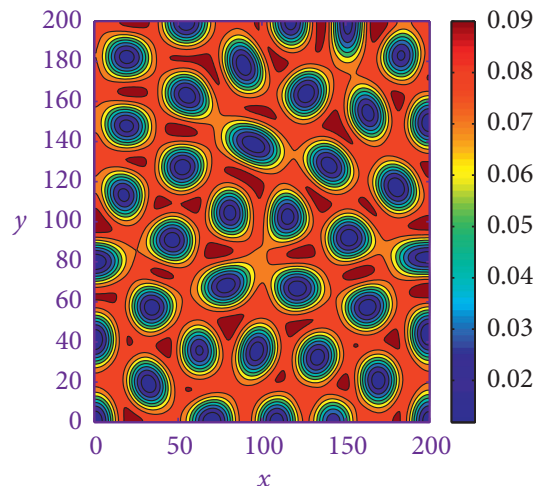

(a)

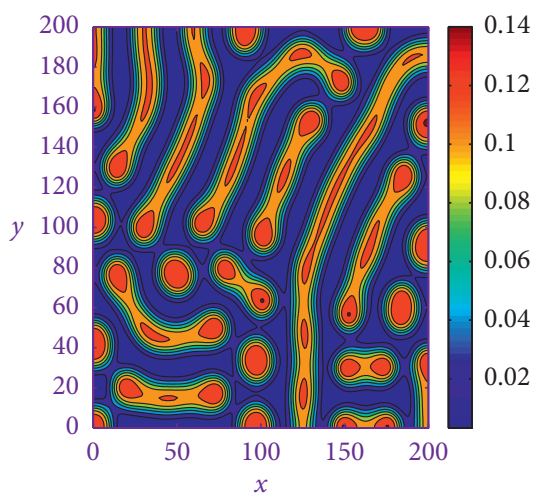

(d)

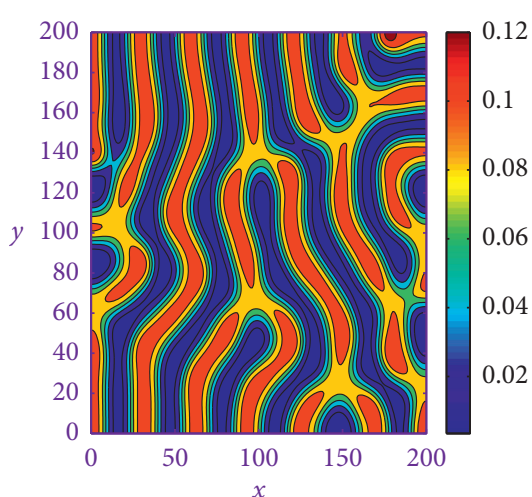

(b)

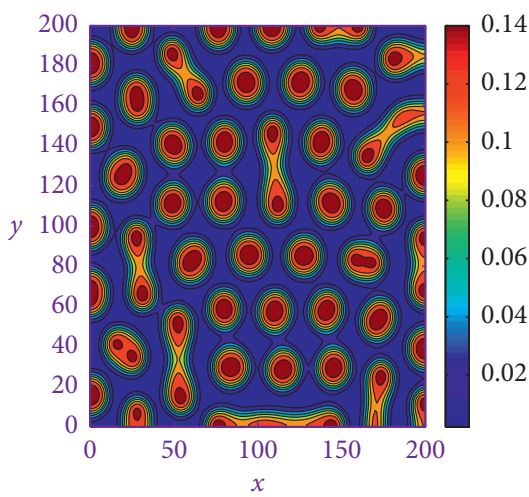

(e)

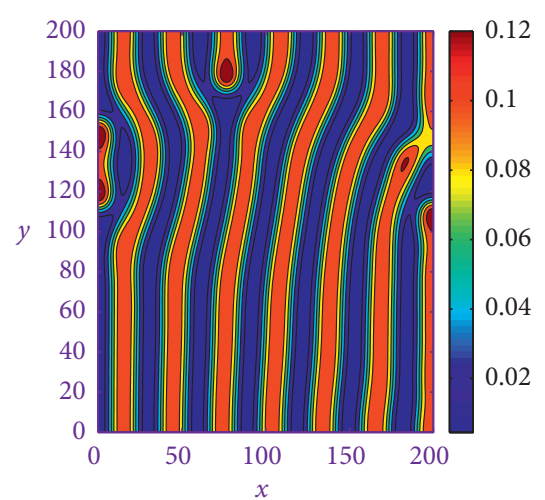

(c)

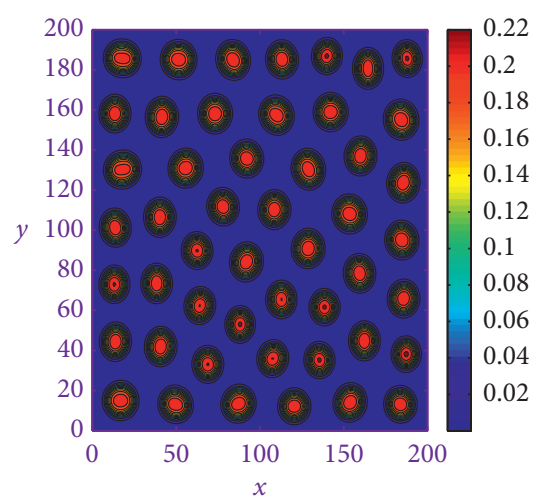

(f)

FIGURE 14: Numerical integration of the reaction-diffusion system (34a)-(34c) with Neumann boundaries; to generate stationary Turing's patterns by prey species (u) at (a) $\delta=0.05$; (b) $\delta=0.053$; (c) $\delta=0.055$; (d) $\delta=0.057$; (e) $\delta=0.06$; and (f) $\delta=0.09$. Other parameter values are $\alpha=0.91881, \beta=0.01, \gamma=0.0327, \varepsilon=0.9, \lambda=0.5, \sigma=0.05, d_{1}=0.1$, and $d_{2}=20.0$.

$$
N=\left(p_{1} u-q_{1}\right) \delta+\left(p_{2} v-q_{2}\right) \sigma=0 .
$$

Since the price and the cost of the predator are not sure, we will consider the following cases in order to determine the bionomic equilibrium:

\section{Case 1}

If

$$
\frac{q_{1}}{p_{1}}>u
$$

i.e.,

$$
p_{1} u-q_{1}<0 .
$$

holds, i.e., to say that the total cost exceeds the total revenue for the harvesting of prey, obviously the prey harvesting will be stopped (i.e., $\delta=0$ ) and the predator harvesting remains operational if $p_{2} v-q_{2}>0$.

Then, from equation (40), we have

$$
v_{1 \infty}=\frac{q_{2}}{p_{2}}
$$

Substituting it in equation (39), we get

$$
\begin{aligned}
u\left(1-u-\frac{\varepsilon v}{\beta+u+\alpha v}\right)= & 0, \\
\Longrightarrow & u=1-\frac{\varepsilon v}{\beta+u+\alpha v}, \\
\Longrightarrow & u=\frac{1}{2}(1-\beta-\alpha v) \\
& \pm \frac{1}{2} \sqrt{1+2 \beta+2 \alpha v+(\beta+\alpha v)^{2}-4 \varepsilon v}, \\
& \Longrightarrow \\
& u_{1 \infty}=\frac{1}{2}\left(1-\beta-\alpha v_{1 \infty}\right) \\
& \pm \frac{1}{2} \sqrt{1+2 \beta+2 \alpha v_{1 \infty}+\left(\beta+\alpha v_{1 \infty}\right)^{2}-4 \varepsilon v_{1 \infty} .}
\end{aligned}
$$




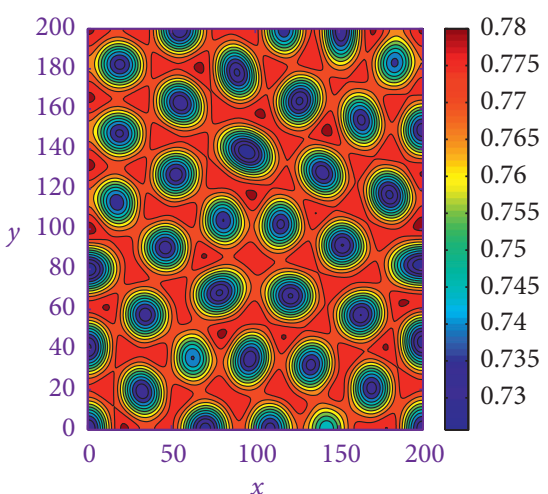

(a)

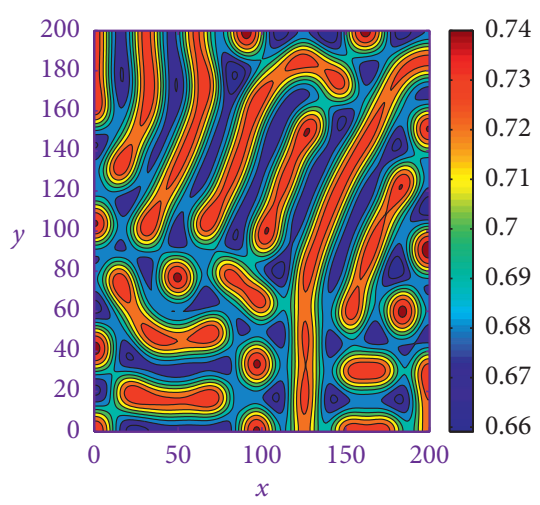

(d)

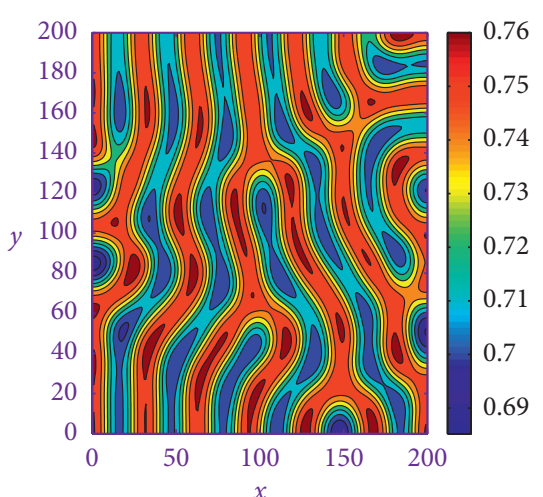

(b)

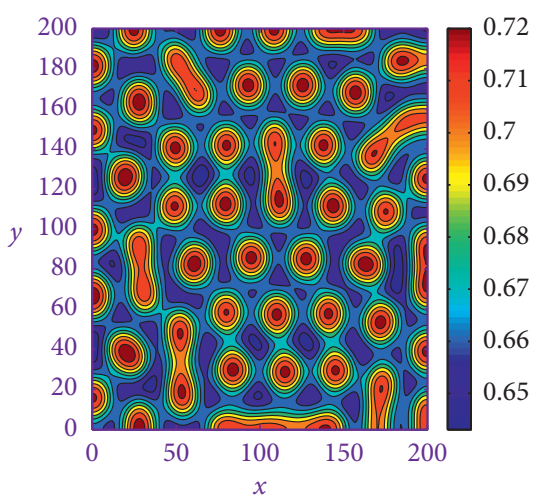

(e)

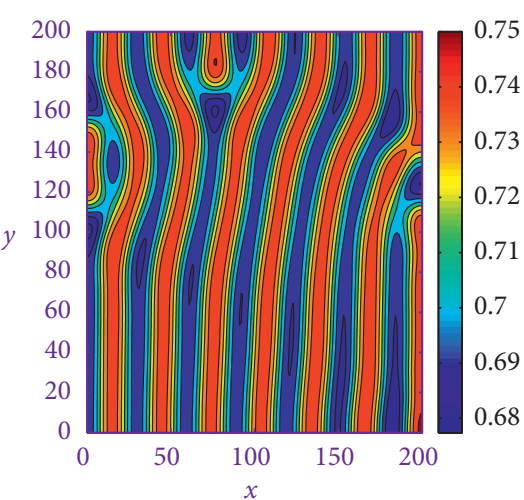

(c)

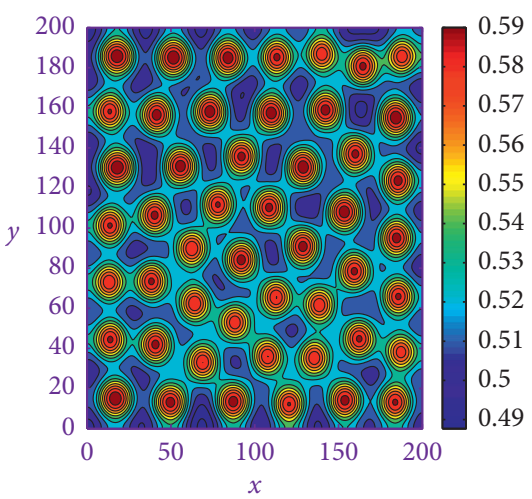

(f)

FIGURE 15: Numerical integration of the reaction-diffusion system (34a)-(34c) with Neumann boundaries; to generate stationary Turing's patterns by predator species $(v)$ at (a) $\delta=0.05$; (b) $\delta=0.053$; (c) $\delta=0.055$; (d) $\delta=0.057$; (e) $\delta=0.06$; and (f) $\delta=0.09$. Other parameter values are $\alpha=0.91881, \beta=0.01, \gamma=0.0327, \varepsilon=0.9, \lambda=0.5, \sigma=0.05, d_{1}=0.1$, and $d_{2}=20.0$.

Again, substituting the value of $u_{1 \infty}$ and $v_{1 \infty}$ in equation (40), we get

$$
\begin{aligned}
\gamma(1-v)+\frac{\lambda u}{\beta+u+\alpha v}-\sigma= & \\
& \Longrightarrow \sigma=\gamma(1-v)+\frac{\lambda u}{\beta+u+\alpha v}, \\
& \Longrightarrow \sigma_{1 \infty}=\gamma\left(1-v_{1 \infty}\right) \\
& +\frac{\lambda u_{1 \infty}}{\beta+u_{1 \infty}+\alpha v_{1 \infty}} .
\end{aligned}
$$

Hence, the following conditions need to be satisfied in order to have the bionomic equilibrium $\left(u_{1 \infty}, v_{1 \infty}, 0, \sigma_{1 \infty}\right)$ : (i) $p_{2}>q_{2}$

(ii) $\gamma>\frac{\gamma q_{2}}{p_{2}}$.

Case 2

If

$$
\begin{aligned}
& \frac{q_{2}}{p_{2}}<v, \\
& \frac{q_{1}}{p_{1}}<u,
\end{aligned}
$$

i.e.,

$$
\begin{aligned}
& p_{2} v-q_{2}>0 \\
& p_{1} u-q_{1}>0
\end{aligned}
$$


holds; in this case, the total revenue exceeds the total cost for two populations and the harvesting is in operational because it can bring profit for capturing species.

From (41), we have

$$
\begin{aligned}
& u_{1 \infty}=\frac{q_{1}}{p_{1}}, \\
& v_{1 \infty}=\frac{q_{2}}{p_{2}} .
\end{aligned}
$$

Substituting the values from (49) in (39) and (40), it is easy to obtain

$$
\begin{aligned}
& \delta_{1 \infty}=1-\left[u_{1 \infty}+\frac{\varepsilon q_{2}}{p_{2}\left(\beta+u_{1 \infty}+\alpha v_{1 \infty}\right)}\right], \\
& \sigma_{1 \infty}=\gamma\left(1-v_{1 \infty}\right)+\frac{\lambda q_{1}}{p_{1}\left(\beta+u_{1 \infty}+\alpha v_{1 \infty}\right)} .
\end{aligned}
$$

So, to have the bionomic equilibrium, i.e., $[u, v, \delta, \sigma] \longrightarrow\left[u_{1 \infty}, v_{1 \infty}, \delta_{1 \infty}, \sigma_{1 \infty}\right]$, the following conditions must be satisfied:

$$
\begin{aligned}
& \text { (i) } 1>u_{1 \infty}+\frac{\varepsilon q_{2}}{p_{2}\left(\beta+u_{1 \infty}+\alpha v_{1 \infty}\right)}, \\
& \text { (ii) } 1>v_{1 \infty}, \\
& \text { (iii) } v_{1 \infty}<\gamma+\frac{\lambda q_{1}}{p_{1}\left(\beta+u_{1 \infty}+\alpha v_{1 \infty}\right)} .
\end{aligned}
$$

It is very much clear that when the intrinsic growth rates of the two species exceed some value, then only the existence of bionomic equilibrium is established.

Example 7. For Case 1, we display trajectories of the solutions of the system (2) with respect to the values $\sigma$ and $\delta$ in Figures 16 and 17, respectively, when $\varepsilon=1.0, \beta=0.031, \delta=$ $0.0, \alpha=0.871881, \gamma=0.0327, \lambda=0.15$ and for Case 2, in Figures 18 and 19, respectively, when $\varepsilon=1.0, \beta=0.0041, \alpha=$ $0.91881, \delta=0.2, \gamma=0.0327, \lambda=0.15$.

5.2. Optimal Harvesting. The one major problem in commercial exploitation of renewable resources is to determine the optical trade-off between present and future harvests. In order to calculate the optimal harvesting policy, we consider the present value $J$ of a continuous time-stream of revenues given by

$$
J=\int_{0}^{\infty} e^{-\omega t}\left\{\left(p_{1} u-q_{1}\right) \delta(t)+\left(p_{2} v-q_{2}\right) \sigma(t)\right\} \mathrm{d} t
$$

where $\omega$ represents the instantaneous annual rate of discount and $\delta$ and $\sigma$ are the control variables. We intend to maximize $J$ subject to the state equations $(2 \mathrm{a})-(2 \mathrm{c})$ by introducing Pontryagin's maximal principle. The Hamiltonian for the problem (52) is given by
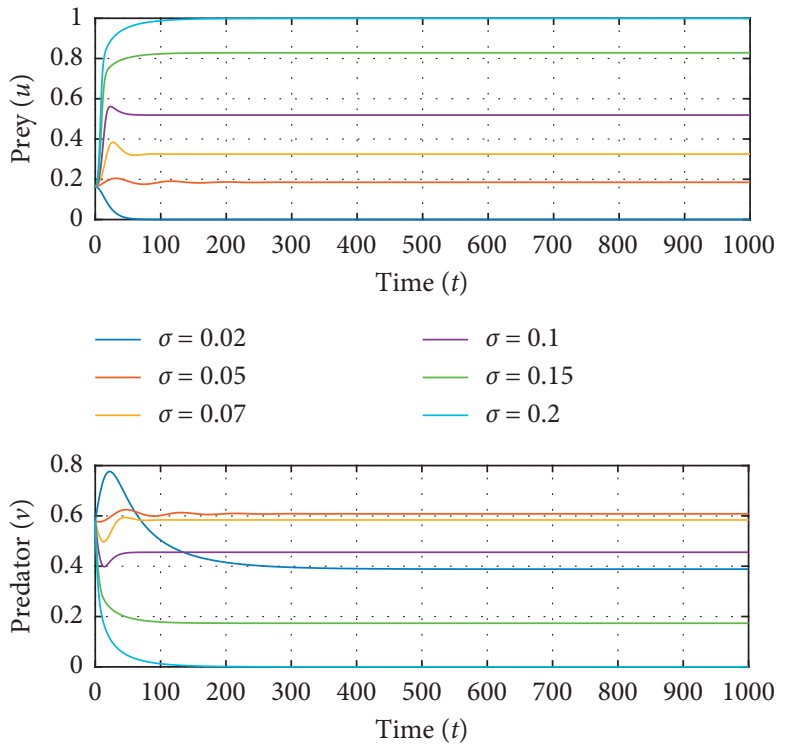

$$
\begin{array}{rlrl}
\sigma & =0.02 \\
\sigma & =0.05 \\
\sigma & =0.07 & -\sigma & =0.1 \\
-\sigma & =0.15 \\
-\sigma & =0.2
\end{array}
$$

Figure 16: Time series plot of the system (2) for Case 1 with different values of $\sigma$ corresponding to the parameter values $\varepsilon=1.0, \beta=0.031, \delta=0.0, \alpha=0.871881, \gamma=0.0327, \lambda=0.15$, and $\left(u_{0}, v_{0}\right)=(0.266,0.278)$.
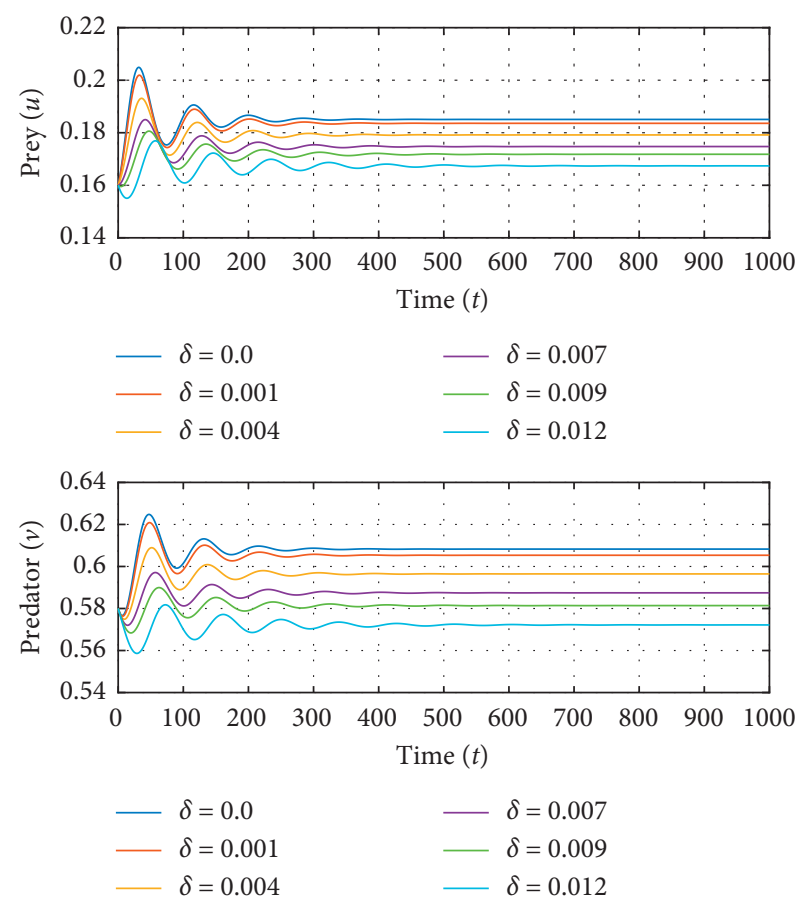

Figure 17: Time series plot of the system (2) for Case 1 with different values of $\delta$ corresponding to the parameter values $\varepsilon=1.0, \beta=0.031, \alpha=0.871881, \sigma=0.05, \gamma=0.0327, \lambda=0.15$, and $\left(u_{0}, v_{0}\right)=(0.266,0.278)$. 

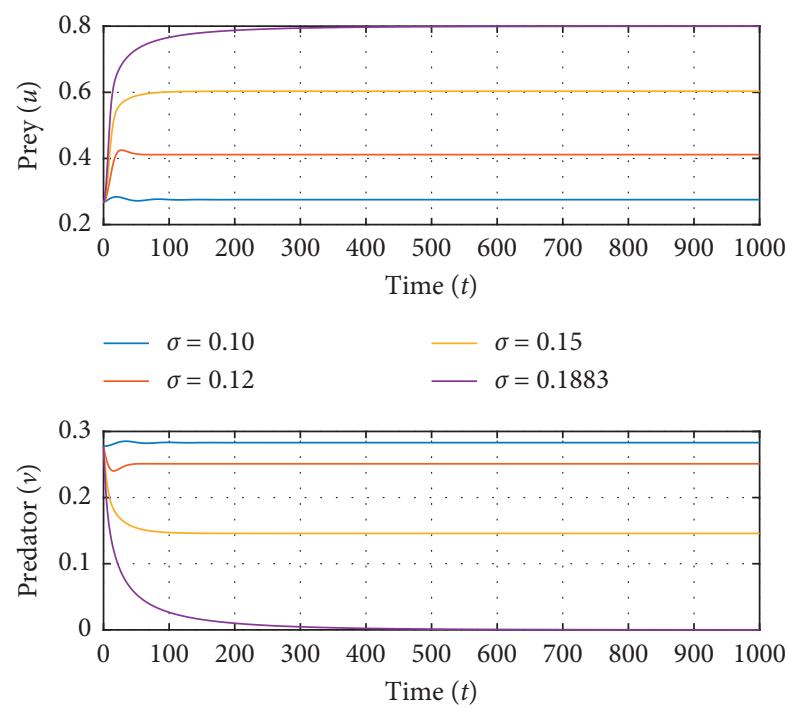

$$
\begin{array}{rlrl}
-\sigma & =0.10 \\
\sigma & =0.12 & -\sigma & =0.15 \\
-\sigma & =0.1883
\end{array}
$$

Figure 18: Time series plot of the system (2) for Case 2 with different values of $\sigma$ corresponding to the parameter values $\varepsilon=1.0, \beta=0.0041, \alpha=0.91881, \delta=0.2, \gamma=0.0327, \lambda=0.15$, and $\left(u_{0}, v_{0}\right)=(0.266,0.278)$.

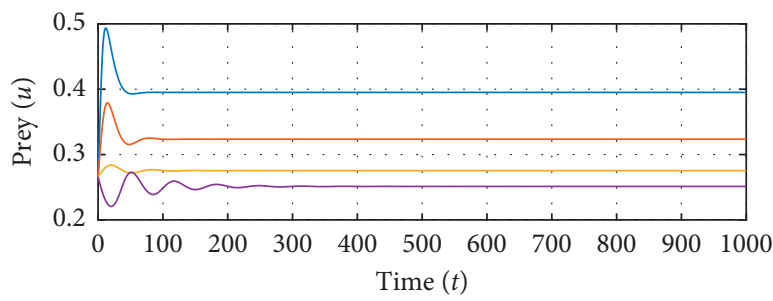

$-\delta=0.10$

$\delta=0.20$

$-\delta=0.16$

$-\delta=0.22$

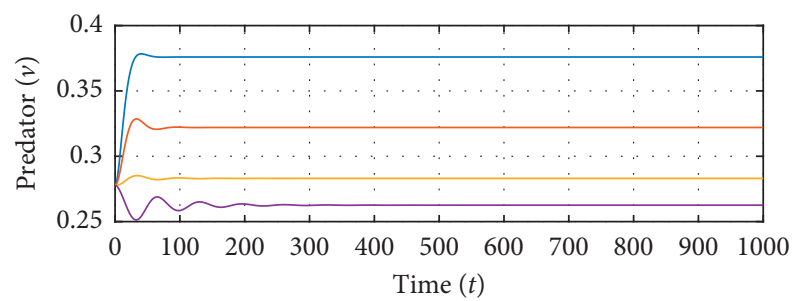

$\delta=0.10$

$\delta=0.20$

$-\delta=0.16$

$-\delta=0.22$

Figure 19: Time series plot of the system (2) for Case 2 with different values of $\delta$ corresponding to the parameter values $\varepsilon=1.0, \beta=0.0041, \alpha=0.91881, \gamma=0.0327, \lambda=0.15, \sigma=0.1$, and $\left(u_{0}, v_{0}\right)=(0.266,0.278)$.

$$
\begin{aligned}
\bar{H}= & e^{-\omega t}\left\{\left(p_{1} u-q_{1}\right) \delta(t)+\left(p_{2} v-q_{2}\right) \sigma(t)\right\} \\
& +\Omega_{1}\left[u(1-u)-\frac{\varepsilon u v}{\beta+u+\alpha v}-\delta u\right] \\
& +\Omega_{2}\left[\gamma v(1-v)+\frac{\lambda u v}{\beta+u+\alpha v}-\sigma v\right],
\end{aligned}
$$

where $\Omega_{1}(t)$ and $\Omega_{2}(t)$ are the adjoint variables.

For optimality, the necessary conditions are

$$
\begin{aligned}
& \frac{\partial \bar{H}}{\partial \delta}=0, \\
& \frac{\partial \bar{H}}{\partial \sigma}=0 .
\end{aligned}
$$

Then, we have

$$
\begin{aligned}
\frac{\partial \bar{H}}{\partial \delta} & =0, \\
e^{-\omega t}\left(p_{1} u-q_{1}\right)-\Omega_{1} u & =0, \\
\Omega_{1} & =e^{-\omega t}\left(p_{1}-\frac{q_{1}}{u}\right) .
\end{aligned}
$$

Similarly,

$$
\Omega_{2}=e^{-\omega t}\left(p_{2}-\frac{q_{2}}{v}\right)
$$

Equations (55) and (56) can also be written as

$$
\begin{aligned}
& \Omega_{1} e^{\omega t}=\left(p_{1}-\frac{q_{1}}{u}\right), \\
& \Omega_{2} e^{\omega t}=\left(p_{2}-\frac{q_{2}}{v}\right) .
\end{aligned}
$$

Therefore, the shadow prices $e^{\omega t} \Omega_{i}(t)$ for $(i=1,2)$ do not vary with time in the optimal equilibrium. Hence, they remain bounded as $t \longrightarrow \infty$ (i.e., they satisfy the transversality condition at $\infty$ ).

We intend to derive here an optimal equilibrium solution of the problem. Since we are considering an equilibrium solution, $u, v, \delta$, and $\sigma$ are to be treated as constants in the subsequent steps.

Now,

$$
\begin{aligned}
\frac{\mathrm{d} \Omega_{1}}{\mathrm{~d} t}= & -\frac{\partial \bar{H}}{\partial u} \\
\Longrightarrow & -\omega e^{-\omega t}\left(p_{1}-\frac{q_{1}}{u}\right)=-e^{-\omega t}\left[\left(p_{1} \delta\right)+\left(p_{1}-\frac{q_{1}}{u}\right)\right. \\
& \cdot\left(1-2 u-\frac{\varepsilon v(\beta+\alpha v)}{(\beta+u+\alpha v)^{2}}-\delta\right)+\left(p_{2}-\frac{q_{2}}{v}\right) \\
& \left.\cdot\left(\frac{\lambda v(\beta+\alpha v)}{(\beta+u+\alpha v)^{2}}\right)\right] .
\end{aligned}
$$


Similarly,

$$
\begin{aligned}
\frac{\mathrm{d} \Omega_{2}}{\mathrm{~d} t}= & -\frac{\partial \bar{H}}{\partial v}, \\
\Longrightarrow & -\omega e^{-\omega t}\left(p_{2}-\frac{q_{2}}{v}\right)=-e^{-\omega t}\left[\left(p_{2} \sigma\right)-\left(p_{1}-\frac{q_{1}}{u}\right)\right. \\
& \cdot\left(\frac{\varepsilon u(\beta+u)}{(\beta+u+\alpha v)^{2}}\right)+\left(p_{2}-\frac{q_{2}}{v}\right) \\
& \left.\cdot\left(\gamma(1-v)-\gamma v+\frac{\lambda u(\beta+u)}{(\beta+u+\alpha v)^{2}}-\sigma\right)\right],
\end{aligned}
$$

From equations (58) and (59), we may find the positive values of $(u, v)=\left(u_{\omega}, v_{\omega}\right)$. follows:

Substituting $\left(u_{\omega}, v_{\omega}\right)$ into (2), we get the equations as

$$
\begin{aligned}
& \delta_{1 \omega}=1-u-\frac{\varepsilon v}{(\beta+u+\alpha v)}, \\
& \sigma_{1 \omega}=\gamma(1-v)+\frac{\lambda u}{(\beta+u+\alpha v)} .
\end{aligned}
$$

Then, the optimal equilibrium efforts levels $\delta_{1 \omega}$ and $\sigma_{1 \omega}$ can be obtained if the following conditions are satisfied:

$$
\begin{aligned}
& \text { (i) } 1>u+\frac{\varepsilon v}{(\beta+u+\alpha v)}, \\
& \text { (ii) } 1>v \text {, with } \gamma v<\gamma+\frac{\lambda u}{(\beta+u+\alpha v)} \text {. }
\end{aligned}
$$

Example 8. If we consider the set of values where $\varepsilon=1.0, \beta=$ $0.041, \alpha=0.91881, \gamma=0.0327, \lambda=0.15, p_{1}=0.5, p_{2}=1.0$, $\delta=0.083, \sigma=0.5$ and $\omega=0.8$, respectively, we can get the optimal equilibrium $\left(u_{\omega}, v_{\omega}\right)=(0.1848452592$, $0.5314707140)$ and the corresponding optimal harvesting efforts are $\delta_{1 \omega}=0.0709708763$ and $\sigma_{1 \omega}=0.05414492630$.

\section{Conclusion}

A large number of interacting species models have been suggested and were explored for a decade to recognize the underlying mechanisms that are happening in real-world ecological systems. In this paper, the dynamical behaviour of a generalist predator-prey model with linear harvesting of each species and Beddington-DeAngelis functional response is totally analyzed. At first, we nondimensionalize the main model, so that it reduces the number of ecological parameters and then the new model (2) experiences for its behavioural studies. In the course of stability theory of ordinary differential equations (ODEs), it has been verified that the interior equilibrium exists under certain conditions and it is globally asymptotically stable via suitable Lyapunov function structure, which signifies that appropriate harvesting has no impact on the persistence property of the harvesting system.
The system under consideration (2) undergoes Hopf and saddle-node bifurcations, with $\varepsilon$ being the bifurcating parameter for both the bifurcations. Subsequently, complete analysis regarding the impact of harvesting is carried out and a conclusion is made that, under some appropriate constraints, harvesting has enormous impact on the final size of the interacting species. Also, the predator harvesting should be greater than prey harvesting (i.e., $\sigma>\delta$ ); if this condition is not fulfilled, then at $(0,0)$ the prey species $(u)$ will meet extinction. Then, for the practical significance, we consider the economic profit of the harvesting. The bionomic equilibrium and optimal harvesting policy are studied. Using Pontryagin's maximum principle, the optimal harvesting policy is discussed. The results show that the optimal harvesting policy may exist and an example is given to show that the optimal harvesting policy of the system (2) is realizable. Finally, our analysis shows that harvesting effort in a generalist predator-prey model plays an imperative role for ecological conservation of interacting species.

A variety of spatiotemporal patterns produced by a Beddington-DeAngelis predator-prey system with reaction-diffusion platform are well studied theoretically and numerically (cf. Figures 10-15). The spatial parametric space is subdivided into stable and unstable regions particularly through Turing's bifurcation curve, as revealed in Figure 9. To generate spatiotemporal patterns, many researchers pointed out that spatiotemporal dynamics of a reaction-diffusion system depend on the choice of initial conditions. Throughout the manuscript, we use a particular type of initial condition for discussing the evolution process of the spatial pattern formation of system $(34 a)-(34 c)[38,39]$. The current investigation points out that the variations of harvesting can additionally show a variety of spatiotemporal patterns for certain range of harvesting of both species. Based on this information, we have concluded that two interacting species reaction-diffusion system with the effect of harvesting can have complex dynamical phenomena containing Turing's patterns scenario.

\section{Data Availability}

The data used in numerical simulation to validate our theoretical study were obtained by using computational tools such as MAPLE and MATLAB.

\section{Conflicts of Interest}

The authors declare that they have no known competing financial interests or personal relationships that could have appeared to influence the work reported in this paper.

\section{Acknowledgments}

The first two authors would like to thank Prof. Santabrata Chakravarty, Department of Mathematics, Visva-Bharati, Santiniketan 731235, India, for his valuable comments that improved the presentation of this research manuscript, and also the first two authors gratefully acknowledge the financial support in part from Special Assistance Programme 
(SAP-III) sponsored by the University Grants Commission (UGC), New Delhi, India (Grant no. F.510/3/DRS-III/2015 (SAP-I)). The third author was supported by the Basic Science Research Program through the National Research Foundation of Korea (NRF) funded by the Ministry of Education (2016R1D1A3B01009330).

\section{References}

[1] A. M. Turing, "The chemical basis of morphogenesis," Philosophical Transactions of the Royal Society of London. Series B, Biological Sciences, vol. 237, pp. 37-72, 1952.

[2] M. Kot, Elements of Mathematical Ecology, Cambridge University Press, Cambridge, UK, 2001.

[3] J. D. Murray, Mathematical Biology, Springer, Berlin, Germany, 2nd edition, 1993.

[4] A. J. Lotka, Elements of Mathematical Biology, Dover Publications, Bureau, IL, USA, 1956.

[5] L. Perko, Differential Equations and Dynamical Systems, Springer, Berlin, Germany, 2013.

[6] R. M. May, Stability and Complexity in Model Ecosystems, Princeton University Press, Princeton, NJ, USA, 2019.

[7] S. J. Schreiber, "Generalist and specialist predators that mediate permanence in ecological communities," Journal of Mathematical Biology, vol. 36, no. 2, pp. 133-148, 1997.

[8] Y. Lv, R. Yuan, and Y. Pei, "Effect of harvesting, delay and diffusion in a generalist predator-prey model," Applied Mathematics and Computation, vol. 226, pp. 348-366, 2014.

[9] L. N. Guin and P. K. Mandal, "Spatiotemporal dynamics of reaction-diffusion models of interacting populations," $A p$ plied Mathematical Modelling, vol. 38, no. 17-18, pp. 44174427, 2014.

[10] L. N. Guin and S. Acharya, "Dynamic behaviour of a reactiondiffusion predator-prey model with both refuge and harvesting," Nonlinear Dynamics, vol. 88, no. 2, pp. 1501-1533, 2017.

[11] P. A. Abrams, "The fallacies of "ratio-dependent" predation," Ecology, vol. 75, no. 6, pp. 1842-1850, 1994.

[12] M. Bohner, M. Fan, and J. Zhang, "Existence of periodic solutions in predator-prey and competition dynamic systems," Nonlinear Analysis: Real World Applications, vol. 7, no. 5, pp. 1193-1204, 2006.

[13] R. Han, L. N. Guin, and B. Dai, "Cross-diffusion-driven pattern formation and selection in a modified Leslie-Gower predator-prey model with fear effect," Journal of Biological Systems, vol. 28, pp. 1-38, 2020.

[14] M. Haque, "Ratio-dependent predator-prey models of interacting populations," Bulletin of Mathematical Biology, vol. 71, no. 2, 14 pages, 2009.

[15] L. N. Guin, B. Mondal, and S. Chakravarty, "Stationary patterns induced by self- and cross-diffusion in a BeddingtonDeAngelis predator-prey model," International Journal of Dynamics and Control, vol. 5, no. 4, pp. 1051-1062, 2017.

[16] T. K. Kar, "Modelling and analysis of a harvested preypredator system incorporating a prey refuge," Journal of Computational and Applied Mathematics, vol. 185, pp. 19-33, 2006.

[17] N. Zhang, F. Chen, Q. Su, and T. Wu, "Dynamic behaviors of a harvesting Leslie-Gower predator-prey model," Discrete Dynamics in Nature and Society, vol. 2011, Article ID 473949, 14 pages, 2011.

[18] L. N. Guin, B. Mandal, and S. Chakravarty, "Existence of spatiotemporal patterns in the reaction-diffusion predatorprey model incorporating prey refuge," International Journal of Biomathematics, vol. 9, no. 6, Article ID 1650085, 25 pages, 2016.

[19] G. Seo and D. L. DeAngelis, "A predator-prey model with a Holling type I functional response including a predator mutual interference," Journal of Nonlinear Science, vol. 21, no. 6, pp. 811-833, 2011.

[20] J. Huang, S. Ruan, and J. Song, "Bifurcations in a predatorprey system of Leslie type with generalized Holling type III functional response," Journal of Differential Equations, vol. 257, no. 6, pp. 1721-1752, 2014.

[21] L. N. Guin, E. Das, and M. Sambath, "pattern formation scenario through turing instability in interacting reaction diffusion systems with both refuge and nonlinear harvesting," Journal of Applied Nonlinear Dynamics, vol. 9, no. 1, pp. 1-21, 2020.

[22] G. Huisman and R. J. De Boer, "A formal derivation of the "Beddington" functional response," Journal of Theoretical Biology, vol. 185, no. 3, pp. 389-400, 1997.

[23] L. Xue, "Pattern formation in a predator-prey model with spatial effect," Physica A: Statistical Mechanics and its Applications, vol. 391, no. 23, pp. 5987-5996, 2012.

[24] M. Haque, "A detailed study of the Beddington-DeAngelis predator-prey model," Mathematical Biosciences, vol. 234, no. 1, pp. 1-16, 2011.

[25] S. Sarwardi, M. Haque, and P. K. Mandal, "Persistence and global stability of Bazykin predator-prey model with Beddington-DeAngelis response function," Communications in Nonlinear Science and Numerical Simulation, vol. 19, no. 1, pp. 189-209, 2014.

[26] J. R. Beddington, "Mutual interference between parasites or predators and its effect on searching efficiency," The Journal of Animal Ecology, vol. 44, no. 1, pp. 331-340, 1975.

[27] D. L. DeAngelis, R. A. Goldstein, and R. V. O’Neill, “A model for tropic interaction," Ecology, vol. 56, no. 4, pp. 881-892, 1975.

[28] J. Sotomayor, "Generic bifurcations of dynamical system$\mathrm{s}^{\dagger \dagger}$ AMS (MOS) 1970 subject classification: 58F99," Dynamical Systems, pp. 561-582, 1973.

[29] P. K. Maini, K. J. Painter, and H. Nguyen Phong Chau, "Spatial pattern formation in chemical and biological systems," Journal of the Chemical Society, Faraday Transactions, vol. 93, no. 20, pp. 3601-3610, 1997.

[30] B. Ermentrout, "Stripes or spots? non-linear effects in bifurcation of reaction-diffusion equations on the square," Proceedings of the Royal Society of London. Series A: Mathematical and Physical Sciences, vol. 434, pp. 413-417, 1991.

[31] B. N. Nagorcka and J. R. Mooney, "From stripes to spots: Prepatterns which can be produced in the skin by a reactiondiffusion system," Mathematical Medicine and Biology, vol. 9, no. 4, pp. 249-267, 1992.

[32] L. Ling and W. Wang, "Dynamics of a Ivlev-type predatorprey system with constant rate harvesting," Chaos, Solitons \& Fractals, vol. 41, no. 4, pp. 2139-2153, 2009.

[33] X. Liu, Y. Liu, and J. Wang, "Bogdanov-takens bifurcation of a delayed ratio-dependent holling-tanner predator prey system," Abstract and Applied Analysis, vol. 2013, Article ID 898015, 5 pages, 2013.

[34] G. Pen, Y. Jiang, and C. Li, "Bifurcations of a holling-type II predator-prey system with constant rate harvesting," International Journal of Bifurcation and Chaos, vol. 19, no. 8, pp. 2499-2514, 2009.

[35] D. Xiao and L. S. Jennings, "Bifurcations of a ratio-dependent predator-prey system with constant rate harvesting," SIAM 
Journal on Applied Mathematics, vol. 65, no. 3, pp. 737-753, 2005.

[36] D. Xiao, W. Li, and M. Han, "Dynamics in a ratio-dependent predator-prey model with predator harvesting," Journal of Mathematical Analysis and Applications, vol. 324, no. 1, pp. 14-29, 2006.

[37] M. Xiao and J. Cao, "Hopf bifurcation and non-hyperbolic equilibrium in a ratio-dependent predator-prey model with linear harvesting rate: Analysis and computation," Mathematical and Computer Modelling, vol. 50, no. 3-4, pp. 360379, 2009.

[38] M. R. Garvie, "Finite-difference schemes for reaction-diffusion equations modeling predator-prey interactions in MATLAB," Bulletin of Mathematical Biology, vol. 69, no. 3, pp. 931-956, 2007.

[39] L. N. Guin and H. Baek, "Comparative analysis between preydependent and ratio-dependent predator-prey systems relating to patterning phenomenon," Mathematics and Computers in Simulation, vol. 146, pp. 100-117, 2018. 